Boise State University

ScholarWorks

Geosciences Faculty Publications and

Presentations

Department of Geosciences

3-2021

\title{
Snow Avalanche Detection and Source Constraints Made Using a Networked Array of Infrasound Sensors
}

\author{
J. B. Johnson \\ Boise State University \\ J. F. Anderson \\ Boise State University \\ H. P. Marshall \\ Boise State University \\ S. Havens \\ Snowbound Solutions LLC \\ L. M. Watson \\ Stanford University
}

This document was originally published in JGR Earth Surface by Wiley on behalf of the American Geophysical

Union. Copyright restrictions may apply. https://doi.org/10.1029/2020JF005741 


\section{JGR Earth Surface}

\section{RESEARCH ARTICLE \\ 10.1029/2020JF005741 \\ Snow Avalanche Detection and Source Constraints Made Using a Networked Array of Infrasound Sensors}

Key Points:

- Infrasound source intensity for a distributed moving source is calculated from a network of arrays

- Video and infrasound observations are jointly used to triangulate the most energetic sources of infrasound

- Flow acceleration over cliff band is responsible for highest sound source intensity

Supporting Information:

- Supporting Information S1

- Movie S1

- Movie S2

- Movie S3

Correspondence to:

J. B. Johnson,

jeffreybjohnson@boisestate.edu

Citation:

Johnson, J. B., Anderson, J. F., Marshall, H. P., Havens, S., \& Watson, L. M. (2021). Snow avalanche detection and source constraints made using a networked array of infrasound sensors. Journal of Geophysical Research: Earth Surface, 126, e2020JF005741. https:// doi.org/10.1029/2020JF005741

Received 9 JUN 2020 Accepted 4 FEB 2021

\author{
J. B. Johnson ${ }^{1}$ (i) J. F. Anderson ${ }^{1}$ (D) H. P. Marshall ${ }^{1}$, S. Havens ${ }^{2}$ (D) and L. M. Watson ${ }^{3}$ (i) \\ ${ }^{1}$ Department of Geosciences, Boise State University, Boise, ID, USA, ${ }^{2}$ Snowbound Solutions LLC, Boise, ID, USA, \\ ${ }^{3}$ Department of Geophysics, Stanford University, Stanford, CA, USA
}

\begin{abstract}
We studied a triggered snow avalanche ( $60 \mathrm{~s}$ in duration and with $\sim 1,100 \mathrm{~m}$ run-out) using a network of infrasound arrays and time-synced video, with the objective of understanding the relationship between infrasound generation and flow dynamics. Using standard array processing techniques, we compared the infrasound source back azimuths with the avalanche flow path identified by frame-differenced, geo-referenced video. Results show that infrasound records begin with direct arrivals followed by echoes from the avalanche-triggering explosions and these decay within $35 \mathrm{~s}$ of the detonations. Subsequent infrasound, which lasts $20-30 \mathrm{~s}$, could then be attributed exclusively to the avalanche. These infrasound detections, and their triangulated source locations, progress downhill over time and the most intense infrasound appears to originate from a steep, mid-path cliff band, where the avalanche reached speeds in excess of $30 \mathrm{~m} / \mathrm{s}$ and accelerations of more than $5 \mathrm{~m} / \mathrm{s}^{2}$. The recorded infrasound was compared to two candidate source models extracted from video: total flow motion and advancing flow motion. Advancing source locations were compared to acoustic intensity time series using a nonnegative least squares inversion to solve for, and to quantify, time-varying infrasound source intensity. We observed that certain portions of the flow, most notably the early stages and the end stages (when the powder cloud was expanding and settling) were infrasonically quiet.
\end{abstract}

Plain Language Summary Rapid gravity-driven flows, such as mud flows, debris flows, and snow avalanches, produce intense infrasound that may be recorded at distances of many kilometers. Infrasound is low-frequency sounds that are inaudible, but travel long distances efficiently and can be recorded using specialized microphones. This study uses eight microphones distributed around a snow avalanche path to map how the avalanche advances and to quantify which part of the snow avalanche produces the majority of the infrasound. We use a video record of the featured avalanche to confirm findings and determine that much of the sound is created as the avalanche accelerates over a steep cliff band. Lessons learned here can be used to better monitor snow avalanche activity elsewhere including highways that are threatened by avalanches following snowstorms.

\section{Introduction}

The detection and study of rapid gravity-driven mass movements using infrasound is a growing area of research and includes studies of hazardous flows like lahars (Johnson \& Palma, 2015), debris flows (Kogelnig et al., 2014; Liu et al., 2015; Marchetti et al., 2019), rockfalls and ice-falls (Johnson \& Ronan, 2015; Preiswerk et al., 2016; Zimmer et al., 2012), pyroclastic density currents (Delle Donne et al., 2014), and iceberg calving (Richardson et al., 2010). Infrasound monitoring has also been proven as an effective tool for identifying the occurrences of snow avalanches and has been implemented as a monitoring strategy both remotely (at tens of kilometers) and proximally (within a few kilometers) using arrays of sensors (e.g., Adam et al., 1998; Arai et al., 2017; Bedard et al., 1988; Comey \& Mendenhall, 2004; Marchetti et al., 2015; Mayer et al., 2020; Ulivieri et al., 2011). Infrasound remote sensing of avalanches may be complemented by seismic monitoring, which must be deployed relatively near the flow path trajectory owing to high seismic wave attenuation in the ground (e.g., Kogelnig et al., 2011; Marchetti et al., 2020). Seismic waves are produced by stresses applied to the ground beneath the flow and also by coupled acoustic waves, which impinge upon and shake the ground locally at the seismometers (Heck et al., 2019).

Infrasonic waves in the air, as opposed to seismic waves in the ground, are recognized as having lower attenuation and superior spatial resolving capabilities for tracking moving avalanches in part due to shorter
(C) 2021. American Geophysical Union. All Rights Reserved. 
wavelengths (e.g., Havens et al., 2014; Johnson et al., 2018; Marchetti et al., 2020). Although single-sensor infrasound recordings may be used to identify large avalanches in low noise conditions, distributed arrays of infrasound sensors have far better detection capabilities (Mayer et al., 2020; Scott et al., 2007). Typically, candidate infrasound source locations are considered at the surface of a topographic model and regions of sound production are then located using intersection of the free surface with array-derived back azimuth and elevation angle constraints (e.g., Marchetti et al., 2020). A natural avalanche in Idaho, for instance was constrained with array back azimuths and known location of the active slide path, permitting estimates of both avalanche descent speeds and slide duration (Havens et al., 2014).

When avalanche path is not known or not visible, a network of arrays can be effective for locating the absolute positions of moving sources. A 3-station network of 4-element infrasound arrays deployed in Little Cottonwood Canyon, Utah has been used to track moving avalanche sources by using a combination of independent array detections and triangulation of their sources through cross-beaming of back azimuths (Johnson et al., 2018). This technique was effective for mapping dozens of natural and triggered avalanches occurring over a season and it built upon lessons learned from an infrasound monitoring network that had been operational in Little Cottonwood, Utah and on Teton Pass, Wyoming since 2006 (Yount et al., 2008). Infrasound is an effective tool for detecting snow avalanches (e.g., Bedard et al., 1988), but is particularly powerful when complemented with multiparametric observations including radar and seismic monitoring (e.g., Kogelnig et al., 2011; Marchetti et al., 2020). The relationship between sound generation and mass movement can also be explored with an integrated approach using infrasound and visual observation made with video cameras.

\section{Experiment and Data Collection}

Data in this study correspond to visual-infrasound observations recorded during an hour-long period of snow avalanche activity triggered by explosions at Fortress Ski Area in Alberta, Canada on March 13, 2018. Beginning at 13:00:00 local time six attempts were made to trigger avalanches using helicopter delivery of ammonium nitrate fuel oil (ANFO). The charges were detonated in a $\sim 0.25 \mathrm{~km}^{2}$ region centered at $50.800^{\circ}$ North and $115.200^{\circ}$ West as part of a television documentary/experiment focused on snow avalanches, which aired as Avalanche: Making a Deadly Snowstorm on BBC2 Horizon in November 2018. At least two of the explosive charges successfully triggered avalanches. These events occurred in the JSP slide path and in the Goat path and both were associated with high signal-to-noise infrasound recordings (Figure 1). The avalanches were also captured with a GoPro Black 5 camera recording with narrow 1080p field-of-view (FOV) video recorded at 60 frames per second. The camera was situated approximately $1900 \mathrm{~m}$ asl and $2 \mathrm{~km}$ from the detonation areas and avalanche starting zone.

The Goat event is analyzed in this study because it is associated with a longer visible slide path than the JSP event and it has higher quality infrasound signal. Two coincident detonations and the entire travel path of the avalanche are visible in the Goat event video (see Movie S1), which is converted to plan view mapped avalanche progression as described in the methods section. The Goat Path avalanche can be considered by the American Avalanche Association as possessing a destructive potential D3 ( 1,000 m path length), but with a relatively small size R2 considering its potential path size (Greene et al., 2016).

Infrasound was collected with a network of microphone arrays that were deployed the day before the experiment. Infrasound was recorded as continuous waveform data at 200 samples per second (Hz) and with 64x gain using three-channel 24-bit DiGOS DataCube loggers. These time series have an accurate timing basis using built-in GPS receivers. Deployed infrasound sensors were infraBSU_vers01 models, which incorporate MEMS-type transducers with a low-cut single pole roll-off at $0.039 \mathrm{~Hz}(26 \mathrm{~s})$ and functionality described in Marcillo et al. (2012). The single-bit resolution on the DataCube logger was $0.083 \mathrm{mPa}$ per count, which is significantly lower than the $2 \mathrm{mPa}$ RMS noise floor estimated for the infraBSU_vers01 microphone in the $1-10 \mathrm{~Hz}$ band.

Each infrasound array consisted of either three sensors (i.e., in Arrays A and C) or two sensors at Array $\mathrm{B}$, which had a defective cable to one of the microphones. All sensors were connected with approximately 20 -m cables to the central logger. Sensor locations relative to the datalogger and to each other were surveyed using compass bearing and a measuring tape such that relative locations are accurate to within 
(a) raw signal recorded at Array A

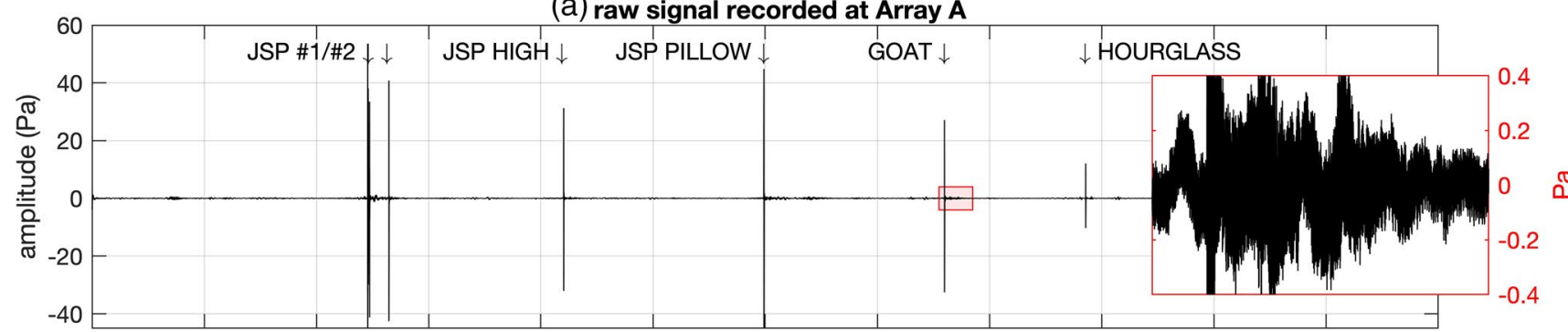

(b) raw spectrogram

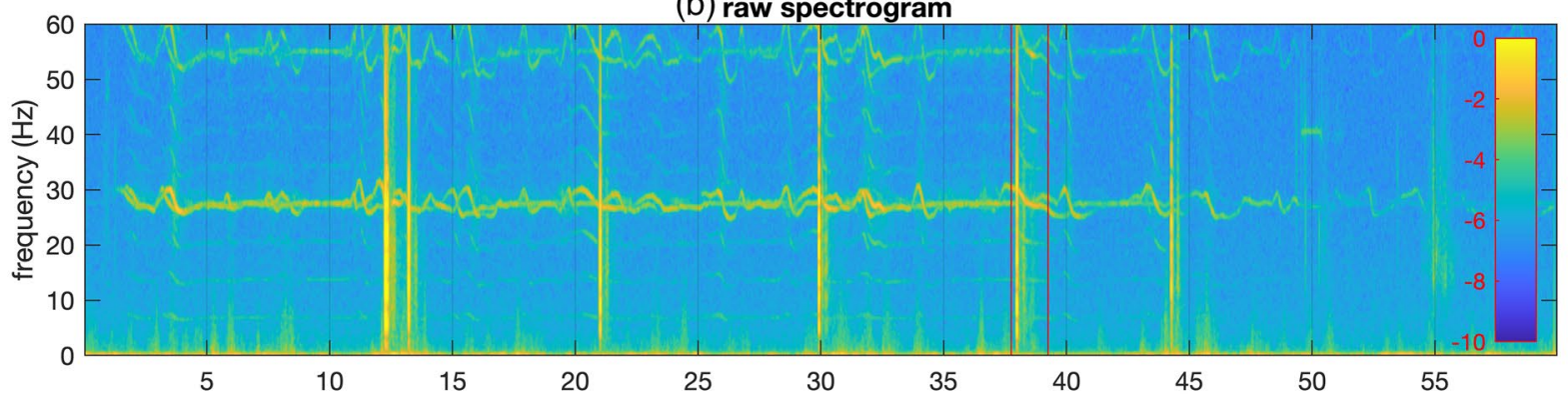

(c) notch filtered signal recorded at Array A

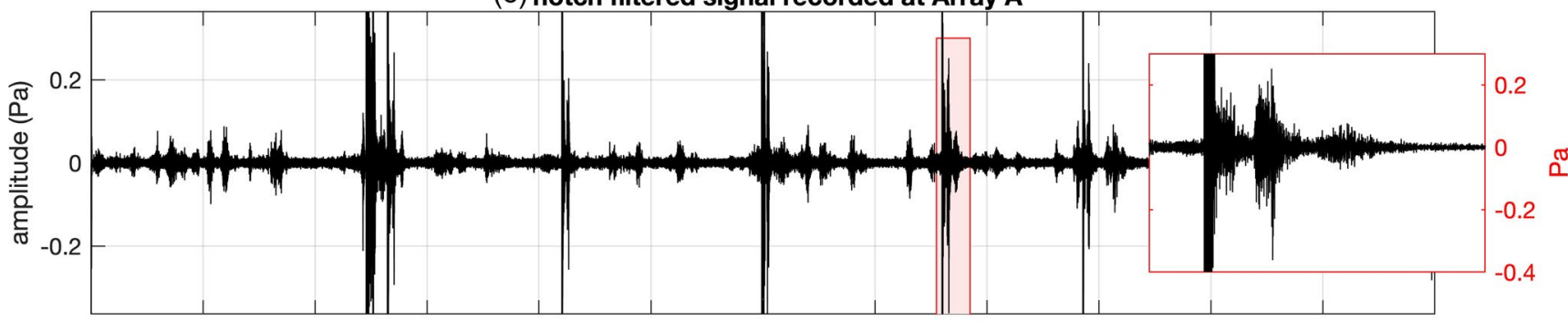

(d) notched spectrogram

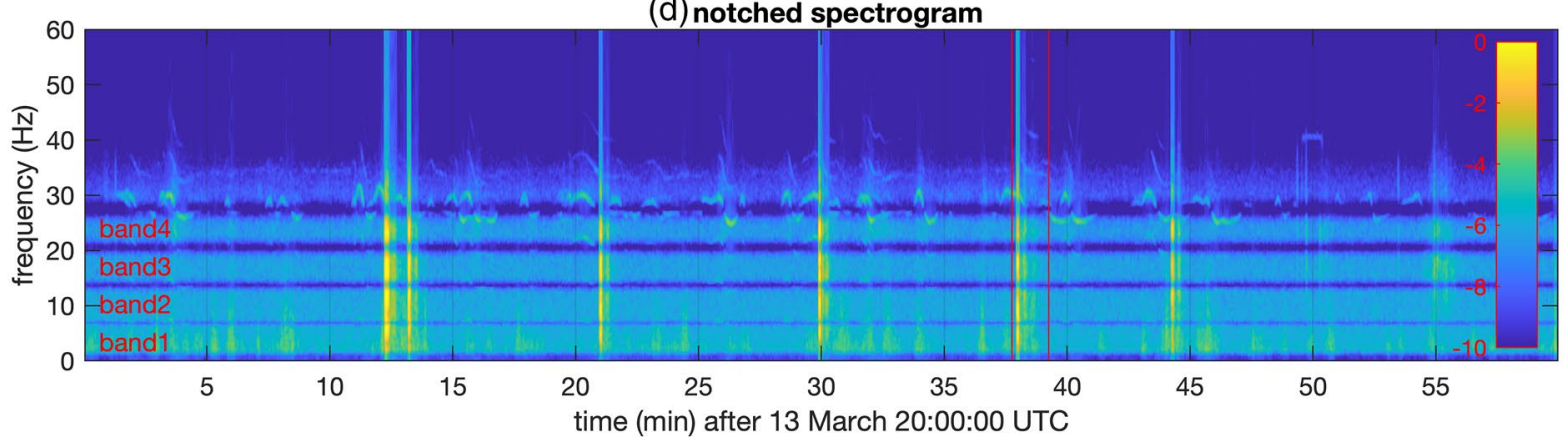

Figure 1. One-hour waveform and power spectrograms for data collected near Fortress Ski Area. (a) Unfiltered infrasound recorded at Array A with detail of the unfiltered event indicated in inset panel. Triggering explosions are named according to slide path and indicated with black arrows; successful triggers occurred for the JSP and Goat slide paths (at 20:13 and 20:38 UTC respectively). (b) Associated power spectrogram for unfiltered data displayed with arbitrary $\log$ scale. The narrow-band frequency peaks associated with integer multipliers of $\sim 6 \mathrm{~Hz}$ correspond to two helicopters flying above the study area. (c) Data displayed with a zoomed-in amplitude scale and with signal band-filtering between 2 and $25 \mathrm{~Hz}$ and with additional notch filters at 6.25-7.5, 12.5-15, 18.7522.5, and 25-30 Hz applied to remove helicopter "noise" (refer to Figure 5). Detail of filtered data for the Goat signal is shown in inset panel. (d) The associated spectrogram for filtered data indicates the four bands that are referenced in later figures and in the text. Spectrogram processing is calculated for $5 \mathrm{~s}$ windows and $4 \mathrm{~s}$ overlap using Hann windows.

$\pm 0.5 \mathrm{~m}$ (Figure 2). Given the approximate 30-m array aperture, possible spatial aliasing and associated back azimuth ambiguity is conceivable for sound wavelengths shorter than $60 \mathrm{~m}$ (i.e., frequencies greater than $\sim 5.5 \mathrm{~Hz}$ ), however spatial aliasing is ameliorated by analyzing broadband avalanche signals across the relatively wide $2-25 \mathrm{~Hz}$ band. Throughout this study we refer to the analyzed signals as infrasound because it 

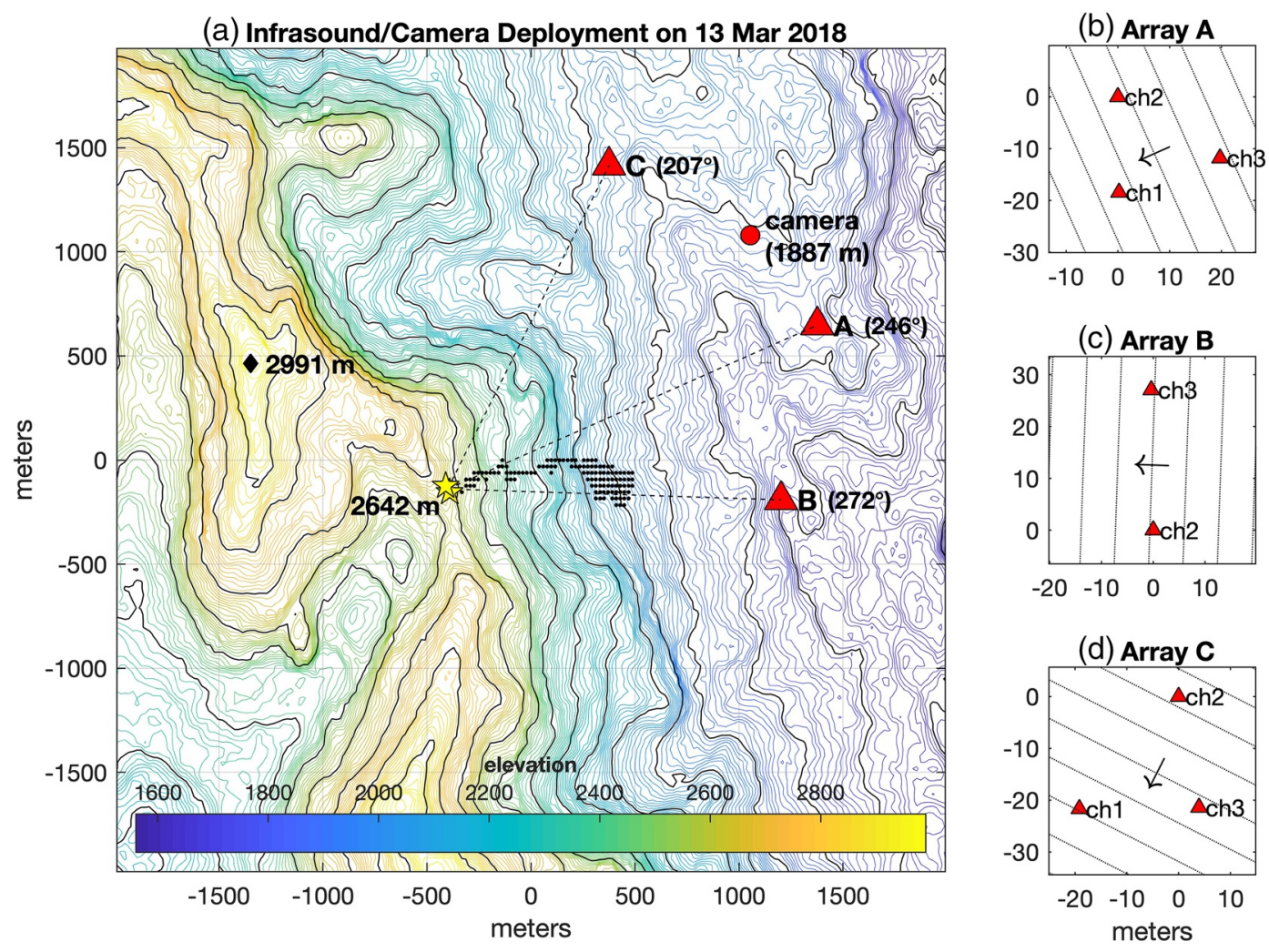

Figure 2. (a) Map centered at $50.800^{\circ} \mathrm{N}$ and $115.200^{\circ} \mathrm{W}$ showing location of video camera and the infrasound arrays. Two coincident explosion sources are indicated with yellow stars and extent of avalanche movement, derived from video data, is indicated with black points plotted at the resolution of the 1 arcsecond DEM (approximately $30 \times 20 \mathrm{~m}$ ). (b-d) Detail of the infrasound array geometries is shown along with back azimuth directions to explosion locations indicated by the arrows. DEM, digital elevation model.

is nearly coincident with the near-infrasound band (1-20 Hz); notably, very little coherent avalanche signal is present in our data in the audible band above $20 \mathrm{~Hz}$ (see Section 3.2).

\section{Methods:}

\subsection{Video Processing}

We converted video data from its original ground-based oblique perspective to plan-view geo-registered imagery to track the progression of the Goat Path avalanche. First, we identified tie points between the video and a digital elevation model (DEM) from the Shuttle Radar Topography Mission (SRTM) 1 arcsecond $(\sim 30 \mathrm{~m})$ resolution map (Figure 3$)$. The four tie points correspond to prominent topography seen in the video and also identified on the DEM. An affine transformation was applied to both geo-register the images properly and to minimize distortion of the GoPro camera lens. The result is that each pixel on a video image frame is associated with a unique elevation angle (relative to horizontal) and to a compass bearing (Figure 3c). Any point within the camera's geo-registered FOV is then back-projected to its intersection with the digital elevation model. The resultant map of video avalanche footage is then used for avalanche tracking and flow quantification. For the Goat event the mapped locations coincide well with expected topographic constraints, which follow a shallow drainage evident in the contour map. The mapped avalanche locations permit comparison of flow progression with infrasound-derived source evolution.

The total spatial extent of the advancing snow avalanche (Figure 4 middle panel) is calculated from frame image differencing using a control frame taken just before the avalanche trigger. The advancing flow extent (Figure 4 left panel) is calculated using differencing of successive image frames. For the advancing flow extent positive difference indicates pixels that were recently perturbed by the moving flow, principally at the 
(a) original image frame

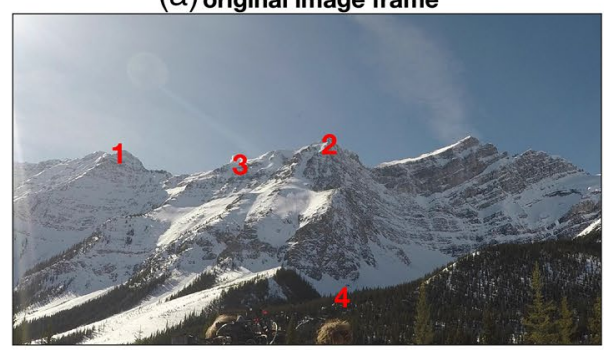

(c) transformed image frame

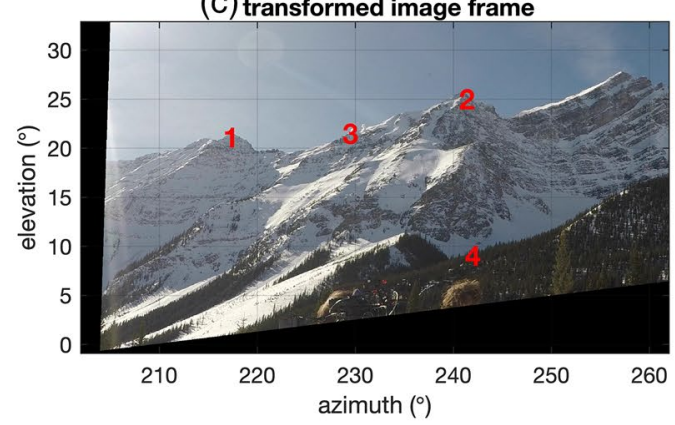

(b) map view tie points

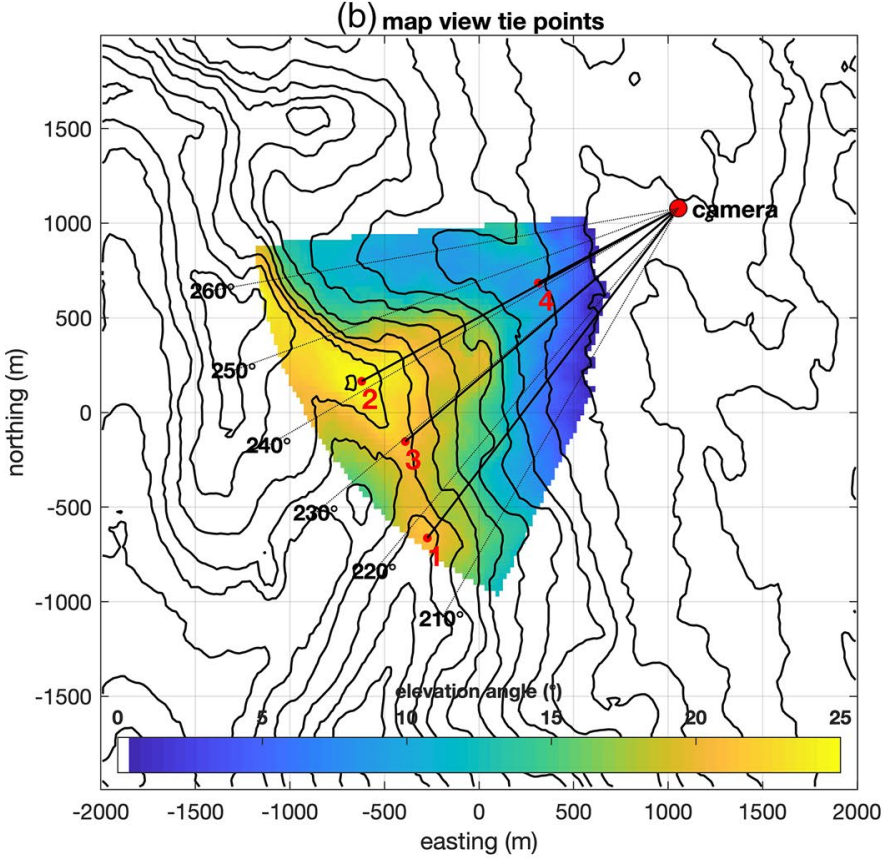

Figure 3. Image-to-map transformation. (a) Image frame showing four tie points that have been identified on (b) the corresponding DEM. Select azimuths and elevation angles (indicated by color bar) from camera to the topography are shown. (c) Registration of the image frames permits mapping of video features to unique azimuth and elevation angles. DEM, digital elevation model.

front of the flow, whereas a negative difference is generally indicative of pixels at the trailing edge of the avalanche and is not shown. The distribution of total flow (TF) area and advancing flow (AF) area can then be used as candidate source region models and compared to infrasound data to gain insight about the source mechanisms and intensity of avalanche infrasound (see discussion Section 5.3).

\subsection{Infrasound Processing}

High signal-to-noise infrasound was recorded at all three arrays for the Goat Path event, however the recorded infrasound includes contributions from avalanche and nonavalanche signal, which originate from a range of directions. These signals include infrasound from two helicopters, the two explosion triggers, and
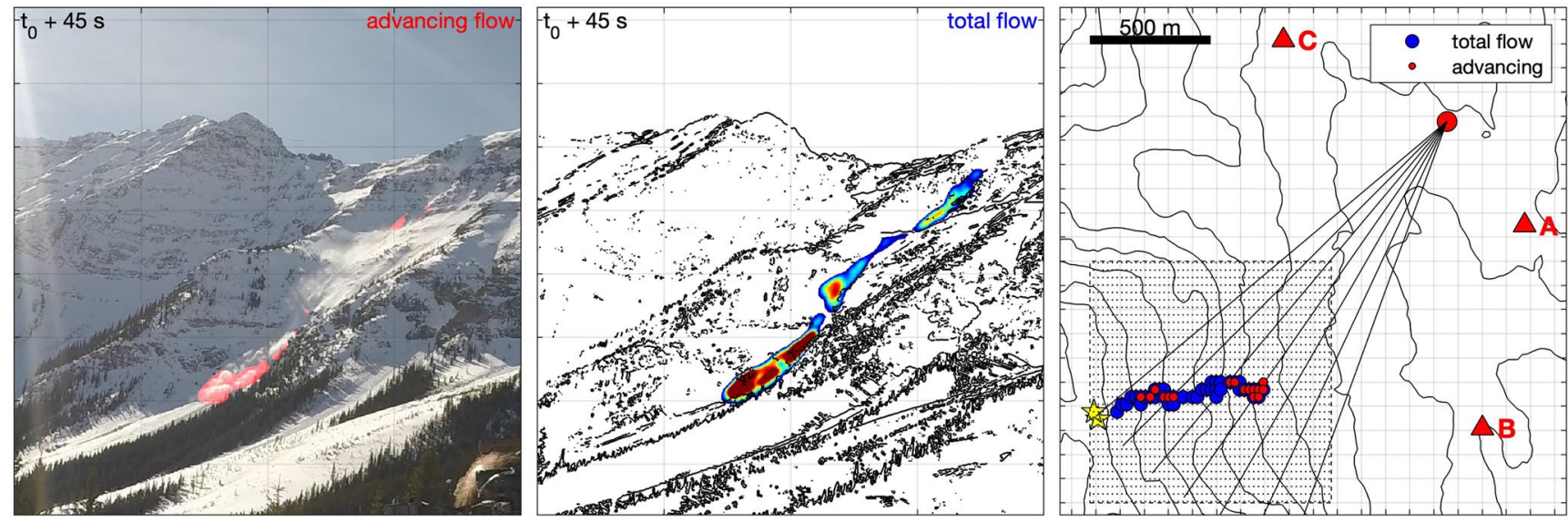

Figure 4. Goat Path avalanche imagery and map shown in term of advancing flow (red highlights in left and right panels) and total active flow (middle panel with colors indicate image difference amplitudes). Equivalent plan view in right panel represents both the total and advancing flow extracted from image differencing over $1 \mathrm{~s}$ intervals. A 90 -s animated sequence of this figure is provided as supporting information (Movie S1). 


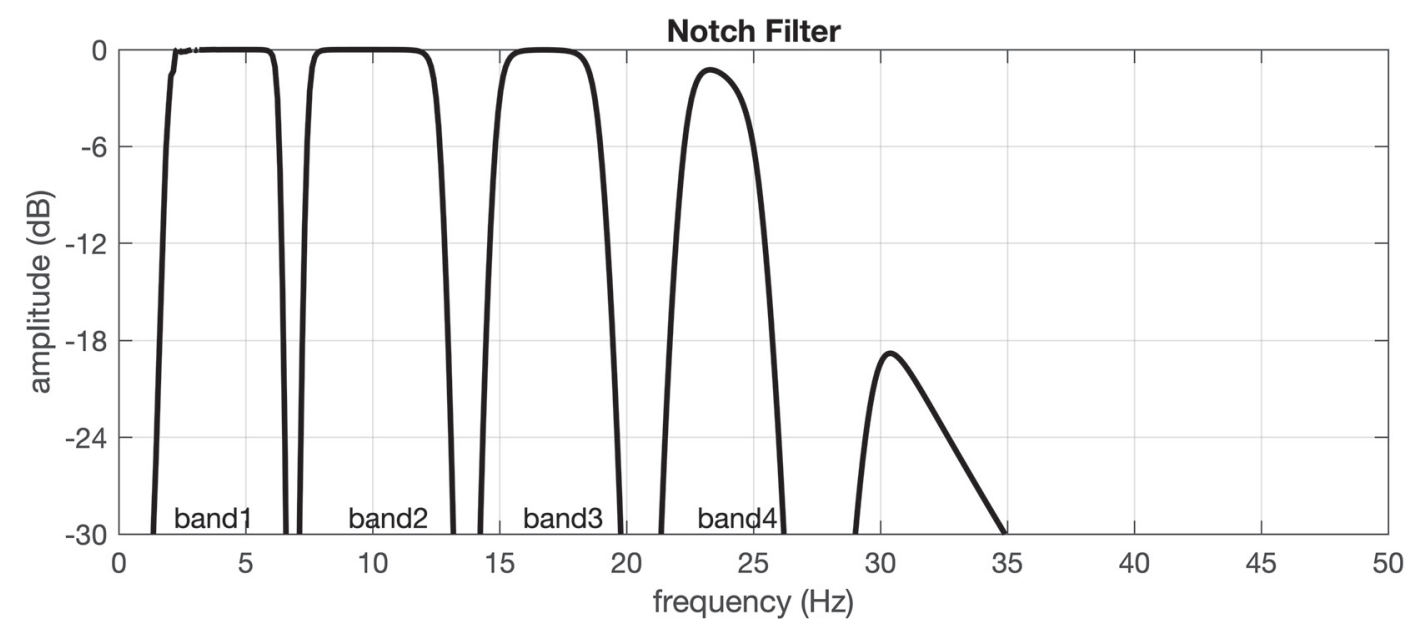

Figure 5. Amplitude spectrum of the multinotch and band pass filter that is applied to the Fortress acoustic data in order to remove helicopter infrasound. Spectrogram and signal after application of this filter is shown in Figure 1 for a 1-h time series and in Figure 6 for a 90-s time series associated with the Goat avalanche event.

explosion echoes off mountain walls, all of which are particular energetic in the infrasound band. Array beam-forming and selective filtering permit identification, isolation, and removal of those signals permitting an analysis of infrasound associated with the avalanche.

\subsubsection{Signal Conditioning and Filtering}

Helicopter infrasound is seen in time series as a sustained infrasonic tremor and is easily recognized in the frequency domain as possessing characteristic spectral peaks corresponding to the rotor angular frequency and its overtones (Stubbs, 2005). The 4-blade Bell helicopters used at Fortress to drop explosives produced infrasound with a fundamental frequency of $6.75 \mathrm{~Hz}$ (corresponding to the rotors' $400 \mathrm{RPM}$ rotation rate), and integer overtones extending at least up to the Nyquist frequency at $100 \mathrm{~Hz}$ (Figure 1b). The most prominent peak averaged over time is at $27 \mathrm{~Hz}$, that is, four times the fundamental frequency.

Helicopter rotor blade angular frequency is relatively constant and the spectrograms illustrate variation in frequencies due to Doppler shift. Because the Doppler frequency shift is modest $(<10 \%)$, it is relatively straightforward to remove helicopter signal using a cascade of notch filters with fixed corner frequencies. We apply a cascade of four four-pole Butterworth IIR stop-band filters at $6.25-7.5 \mathrm{~Hz}, 12.5-15 \mathrm{~Hz}$, 18.75-22.5 Hz, and 25-30 Hz in addition to a fourth-order bandpass filter between 2 and $25 \mathrm{~Hz}$. Without these filters, the avalanche infrasound is overwhelmed by nonnatural incident sound and avalanche signal analysis is not feasible. The additional bandpass filter $(2-25 \mathrm{~Hz})$ is designed to coincide with the expected band of infrasound associated with snow avalanches of similar size occurring in other regions (e.g., Havens et al., 2014; Johnson et al., 2018; Marchetti et al., 2015). The cumulative filter is represented in a Bode amplitude response plot (Figure 5).

The notch filters are used to define frequency bands referred to as \#1 (2-6.25 Hz), \#2 (7.5-12.5 Hz), \#3 $(15-18.75 \mathrm{~Hz})$, and \#4 (22.5-25 Hz). These band limits are also used to quantify infrasound intensity $\left(\mathrm{W} / \mathrm{m}^{2}\right)$ recorded at each station in discrete frequency bands, which is useful for articulating the most energetic portion of the avalanche infrasound spectrum. Infrasound intensity ( $I$ ), or received acoustic power density, is related to the root mean squared excess pressure divided by the atmospheric impedance

$$
I=p^{2} / z
$$

where $p$ denotes the peak pressure of a sustained signal and impedance $z$ is the product of sound speed and atmospheric density ( $\sim 00 \mathrm{~Pa} \mathrm{~s} / \mathrm{m}$ at the elevation of the study area). Infrasound intensity time series are calculated as the average values from the multiple microphone elements in a given array and smoothed using a 5-s running average, which conserves total energy. Infrasound intensity time series are also normalized by the width of the frequency bands (i.e., \#1: $4.25 \mathrm{~Hz}$; $\# 2: 5 \mathrm{~Hz}$; \#3: $3.75 \mathrm{~Hz}$; 4: $2.5 \mathrm{~Hz}$ ) and displayed in units of $\mathrm{W} / \mathrm{m}^{2} / \mathrm{Hz}$ (e.g., Figures $6 \mathrm{c}-6 \mathrm{~g}$ ). It is notable that the infrasound intensity varies over many orders of 
(a) Goat avalanche infrasound

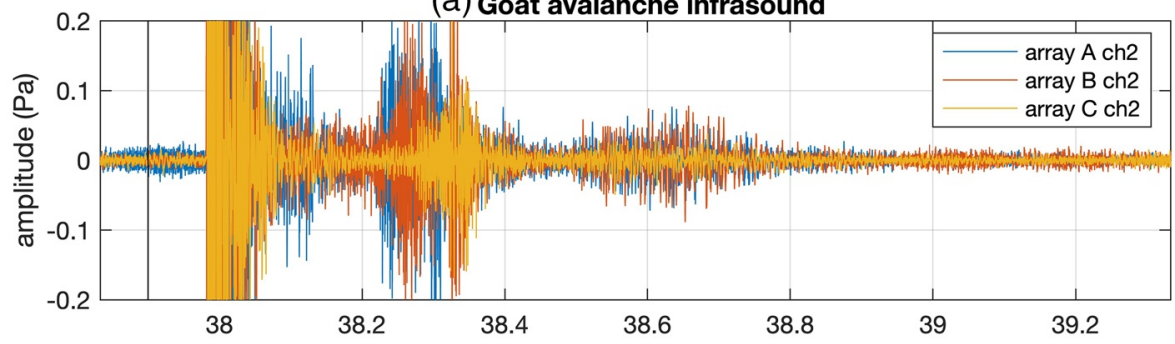

(b) Array B spectrogram with notch filter applied

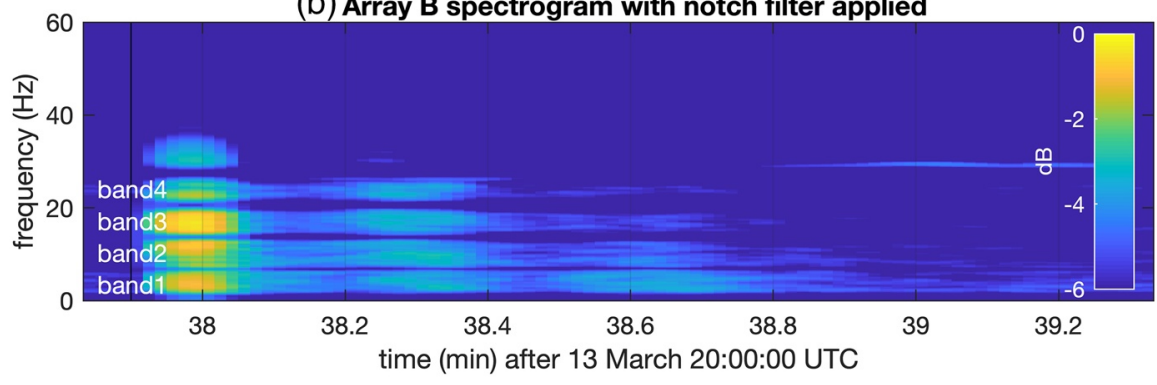

(c) band 1 intensity $(2.0-6.25 \mathrm{~Hz})$
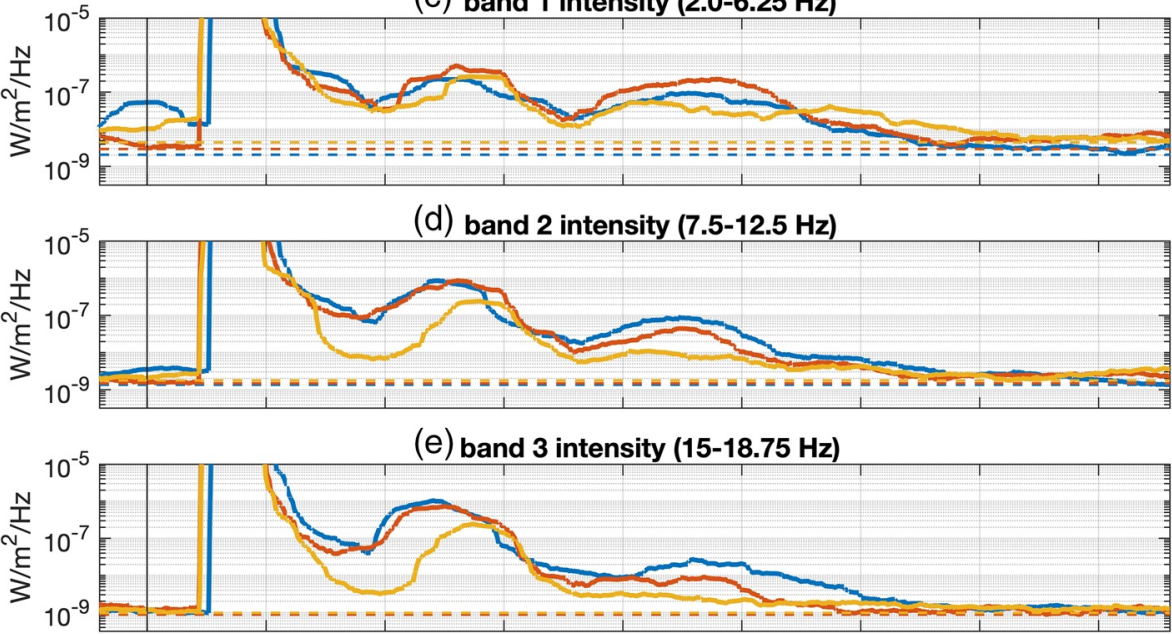

(f) band 4 intensity $(22.5-25 \mathrm{~Hz})$
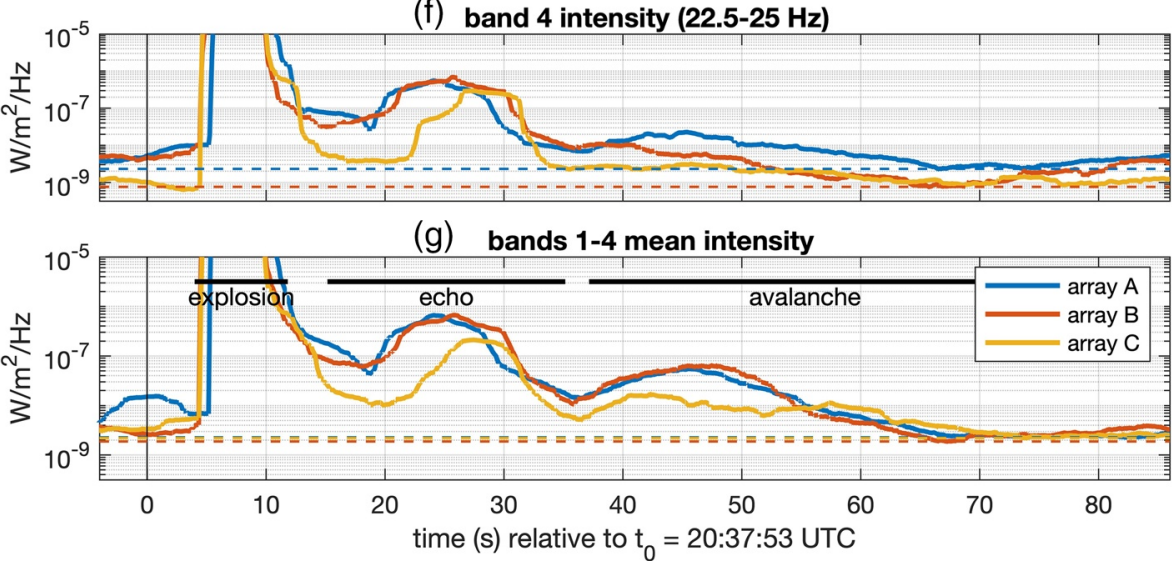

Figure 6. Goat avalanche infrasound detail. (a) Goat infrasound time series after application of notch filter described in Figure 5 and (b) its spectrogram calculated for 5-s time-centered windows. (c-g) Power densities (intensities) for the band-limited signals smoothed over $5 \mathrm{~s}$ windows. Approximate noise levels for each array and each band are indicated with dashed horizontal lines. In (g), the sources of sound, including direct avalanche, echoes, and direct avalanche, are annotated. 
magnitude for the explosions, echoes, and avalanche signal, thus logarithmic intensity scales are particular useful for comparing the different source energetics and identifying noise floors, which we quantify from the postcoda minimum amplitude values.

The recorded explosion and echo infrasound have higher intensity than the avalanche signal (Figure 6g). The echo power spectral density appears fairly constant through all frequency bands, though a subtle dropoff in the higher frequency bands is evident for Array C located slightly farther from the echoes than Arrays $\mathrm{A}$ and $\mathrm{B}$. For the avalanche signal beginning about $t_{0}+35 \mathrm{~s}$ (where $t_{0}$ is the explosion time), the energy is not constant over all bands. Infrasound energy density appears most pronounced in bands \#1 and \#2 (below $12.5 \mathrm{~Hz}$ ) suggesting that this is a critical frequency band for both avalanche detection and location mapping (described in Section 4).

\subsubsection{Array Processing}

The origin of infrasound sources, including explosions, explosion echoes, and avalanche signal are inferred from array analysis summarized below and in Table 1. Standard array beam forming analysis is applied to $5 \mathrm{~s}$ time windows with $4 \mathrm{~s}$ overlap. Our array processing workflow, which allows determination of signal back azimuth, is enumerated as follows:

1) Data are filtered using the multinotch, band-pass filter described above in signal conditioning

2) Cross correlation functions are calculated among channels within the three arrays

3) Cross-correlation results are then upsampled, smoothed, and displayed as correlograms (Figure 7)

4) High-quality lag times for maximum cross correlation amplitudes are identified

5) Lag times are used to calculate back azimuth slowness vectors

6) Slowness vectors are converted to back azimuths and elevation angles

Table 1 elaborates on these analytical techniques for 3-sensor and 2-sensor configurations, whose processing is handled slightly differently in steps 4-6. We note that the use of a two-element "array" is nontraditional, however two sensors can still prove effective for back azimuth determination when the general direction of infrasound incidence may be assumed (e.g., it is coming from the direction of the avalanche slope).

\subsection{Infrasound and Camera Synchronization}

The slowness vectors associated with each coherent-energy time window are converted to back azimuths of incident infrasound as described in Table 1 step 5. For all arrays the sounds arriving 6-10 s after the visible explosion have back azimuths closely associated with the expected direction to the detonations $\left(246^{\circ}\right.$, $207^{\circ}$, and $272^{\circ}$ ). Given their compact (point source) nature and high amplitude, signals from the two avalanche-triggering detonations are also locatable using arrival time triangulation (yellow stars in Figure 2 map) using the timing $t_{i}$ of peak pressure associated with the explosion pulses (Figure 8). Three arrival times at the three arrays may then be used to locate the source using a grid-search. We find the source origin time and latitude and longitude of the two sources assuming that the explosions are fixed to the DEM surface, that is, $z=\operatorname{DEM}(x, y)$, and that propagation from source to receiver is nearly line-of-sight:

$$
\operatorname{RES}^{2}\left(x, y, t_{0}\right)=\sum_{i=A, B, C}\left(\frac{\sqrt{\left(z-z_{i}\right)^{2}+\left(x-x_{i}\right)^{2}+\left(y-y_{i}\right)^{2}}}{c}-\left(t_{i}-t_{0}\right)\right)^{2}
$$

Optimal source parameters for the explosions are found by minimizing the residual in Equation 2. Source accuracy can be affected by uncertainties in the speed of sound, $c$, which depends mainly upon atmospheric temperature, but we are able to solve for an appropriate speed of sound that gives rise to mapped explosion locations that are validated by the video. Coincidence of projected explosion sources (derived from mapped video) and infrasound-derived locations occur when sound speed $c=332 \mathrm{~m} / \mathrm{s}$, which is associated with a reasonable temperature of $0^{\circ} \mathrm{C}$. The source time $t_{0}$ is then calculated as 20:37:53.5 $\pm 0.1 \mathrm{~s}$ for the two events. We can use this explosion time to precisely synchronize the GoPro video frames. Video-derived timing of the two explosions show occurrence within two video consecutive frames, meaning that both detonations occur nearly coincidentally, within $0.03 \mathrm{~s}$. 
Table 1

Infrasound Workflow Processing Steps From Signal Conditioning to Back Azimuth Computation

Workflow step Details

$1 \quad$ Pressure data are filtered using multinotch band-pass IIR filter.

2 Cross-correlation analysis for all array elements is performed for overlapping time windows.

3

Cross-correlation results are upsampled and smoothed (Figure 7)

4a (A/C) Well correlated and consistent time windows are considered candidates for infrasound signals

$4 \mathrm{~b}$ (B) Well correlated and reasonable lag times are considered candidates for infrasound signals

5a (A/C) Lag times are used to calculate a unique back azimuth defined by a slowness vector

$5 \mathrm{~b}$ (B) Lag times are used to determine two candidate slowness vectors for an assumed elevation angle of infrasound crossing the array

6a (A/C) Slowness vector is converted to elevation angle and back azimuth

6b (B) Slowness vector is converted to back azimuth
$Y[f]=P[f] \times H[f]$ where $P[f]$ is the complex frequency spectrum of $p(t)$ and $H[f]$ is the complex filter, whose amplitude response is indicated in Figure 5.

Three comparisons are made for Arrays $\mathrm{A}$ and $\mathrm{C}$ and one comparison is made for Array B (which has only two channels). Five second Hann windows are analyzed at one second time steps (i.e., with $4 \mathrm{~s}$ overlap).

Resampling with cubic interpolation (by factor of 5) has been applied to each column of the correlograms shown in Figure 7. A Gaussian smoothing filter has also been applied to all rows using a standard deviation of $1 \mathrm{~s}$. This serves to weight the lag values by adjacent lag times.

Windowed data are considered signal if mean normalized cross-correlation values exceed a certain level, in this case 0.5. For Arrays A and C, a consistency threshold is also applied that is the lag time of the triad pairs must approach zero (e.g., Cansi, 1995): $\left|\delta t_{21}+\delta t_{32}+\delta t_{13}\right| \leq 0.015 \mathrm{~s}$.

For Array B, which had only two elements, the window is considered signal if normalized cross-correlation values exceed 0.5 and if correlated lag time is less than the maximum slowness crossing the array. So, with sensors separated by $\sqrt{\delta x^{2}+\delta y^{2}+\delta z^{2}}=27 \mathrm{~m}$, the maximum expected lag time for $c=331 \mathrm{~m} / \mathrm{s}$ is $0.082 \mathrm{~s}$.

For Arrays A and C, the slowness vector, $\vec{S}=\left(s_{x}, s_{y}, s_{z}\right)$ is found by inversion of: $\left[\begin{array}{ll}\delta d_{x 21} & \delta d_{y 21} \\ \delta d_{x 32} & \delta d_{y 32} \\ \delta d_{x 13} & \delta d_{y 13}\end{array}\right]\left[\begin{array}{l}s_{x} \\ s_{y}\end{array}\right]=\left[\begin{array}{l}\delta t_{21} \\ \delta t_{32} \\ \delta t_{13}\end{array}\right]$, where $s_{z}=\sqrt{1 / c^{2}-s_{x}^{2}-s_{y}^{2}}$

For Array B, the horizontal slownesses are calculated as two solutions to the quadratic equation and for horizontal slowness amplitude, which is the inverse of the apparent velocity, that is, $c_{a}=c \cos \varphi$. We fix $\varphi=10^{\circ}$ in this analysis accounting for the estimated elevation angle from slide path to sensing arrays. Noting the sign of the radical term, the two solutions are then $s_{x}=\frac{\delta t \delta x \pm \delta y \sqrt{\left(\delta x^{2}+\delta y^{2}\right) / c_{a}^{2}-\delta t^{2}}}{2\left(\delta x^{2}+\delta y^{2}\right)}$
and $s_{y}=\frac{\delta t \delta y \mp \delta x \sqrt{\left(\delta x^{2}+\delta y^{2}\right) / c_{a}^{2}-\delta t^{2}}}{2\left(\delta x^{2}+\delta y^{2}\right)}$ The unrealistic slowness vector can be discarded if, for instance, it originates from an unanticipated direction.

The back azimuth $\theta=a \tan 2\left(s_{x} / s_{y}\right)$; elevation angle $\varphi=a \tan \left(s_{z} / \sqrt{s_{x}^{2}+s_{y}^{2}}\right)$.

The back azimuth $\theta=a \tan 2\left(s_{x} / s_{y}\right)$; elevation angle $\varphi=10^{\circ}$ is fixed in this analysis. 

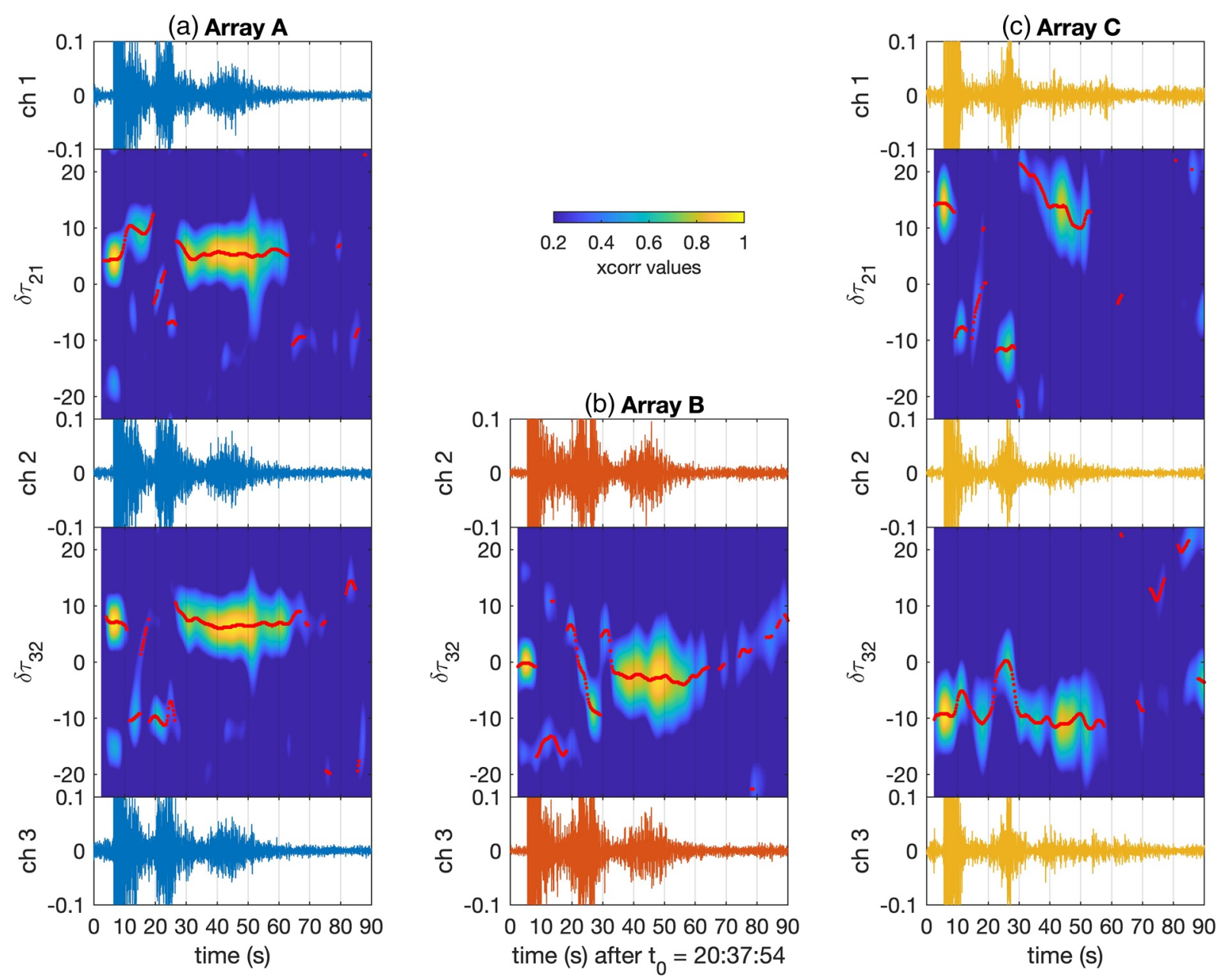

Figure 7. Filtered waveform data and normalized cross-correlation "correlograms" for overlapping $5 \mathrm{~s}$ time windows given for (a) Array A, (b) Array B, and (c) Array C. Red dots in the correlograms indicate those time windows for which lag times are considered both reliable (refer to step 4 of Table 1) and also associated with infrasound signal crossing the arrays. Lag times of correlograms are in units of samples (one sample $=0.005 \mathrm{~s}$ ) and waveform amplitudes are in $\mathrm{Pa}$.

\section{Results}

\subsection{Infrasound Detection and Back Azimuth Evolution}

Substantial infrasound energy between 17 and $33 \mathrm{~s}$ after the explosions (at $t_{0}$ ) is detected on all three stations and originates from a broad sweep of azimuths ranging from the north to the east (nearly opposite of the direction to the explosion and slide path). These back azimuth directions point to steep valley walls $4-5 \mathrm{~km}$ from the explosions and located about 2 or $3 \mathrm{~km}$ from the various arrays using estimated back projection. Two-way travel distances of 6-8 km ( 18-24 s travel times) is consistent with infrasound echoes produced by the initial explosions reflecting off these distant ridges. The extended time duration of the echoes (tens of seconds), and range of its back azimuths (Figure 9), points to multiple reflections from the complex mountain topography.

Infrasound echoes taper off at about $t_{0}+33 \mathrm{~s}$ and are soon replaced by a new pulse of infrasound that continues for several tens of seconds on all three arrays. This signal is associated temporally with active movement of the avalanche and their array back azimuths point to the Goat slide path for all three arrays. Each array appears to detect signal from Goat beginning at around similar times $\left(t_{0}+\sim 27-36 \mathrm{~s}\right)$, but detections continue for different lengths of time at the different arrays (Figure 9b). For instance, Array C, which is furthest from the slide path senses the avalanche until about $53 \mathrm{~s}$, when it appears to be obscured 


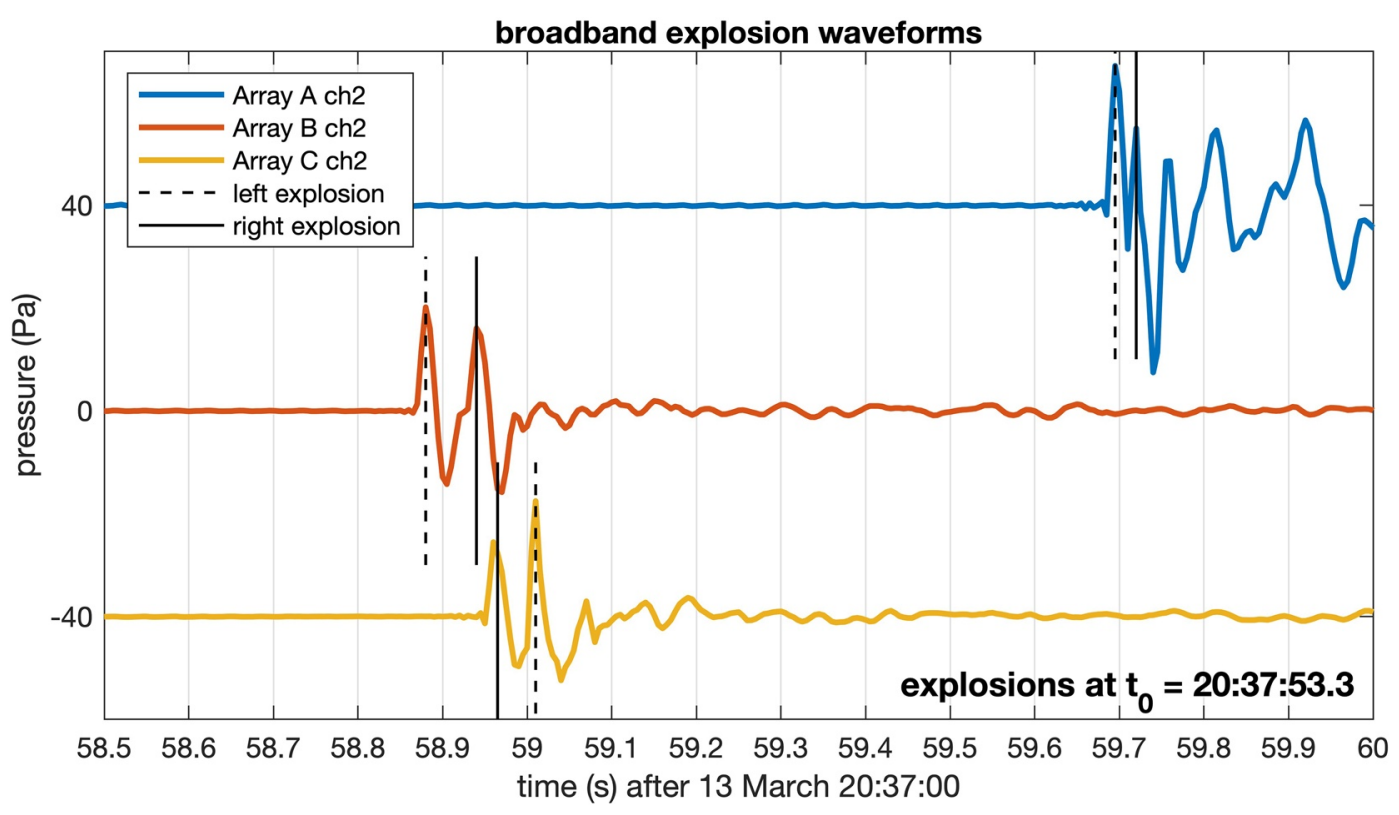

Figure 8. Broadband $(>0.1 \mathrm{~Hz})$ explosion waveforms recorded on channel 2 of the three arrays. Timing of peaks are shown for the two explosions, which are labeled "left" and "right" in terms of how they are viewed from the camera.

by helicopter sounds at around $55 \mathrm{~s}$. Array A is sensitive to the avalanche signal out to around $64 \mathrm{~s}$, while Array B (closest to the runout of the Goat slide path) appears to pick up avalanche infrasound (albeit with diminished intensity) out to at least $t_{0}+65 \mathrm{~s}$.

In general, moving sources that occur near infrasound arrays manifest with changing back-azimuths. These changing source directions may be used to track moving sources for signals confined to an assumed path (e.g., Delle Donne et al., 2014; Johnson \& Palma, 2015; Johnson \& Ronan, 2015), however when precise path orientation is unknown two or more arrays may be used to triangulate sound generation sources for both fixed and moving sources (e.g., Arrowsmith et al., 2012; Johnson et al., 2018). The triangulation of sources using multiple beams is improved when the arrays are distributed close to, and around, the sources. In the case where arrays are too far separated, there is the possibility that each array is sensing different parts of a distributed flow and back azimuths will not clearly coincide. Network localization of a single distinct source may not be possible and the assumption of a single point source of infrasound production (e.g., Marchetti et al., 2020) might not be valid for all snow avalanches.

\subsection{Infrasound Localization of Sources Using Multiple Arrays}

From video camera observations, the full geographic extent of the Goat Slide path can be reconstructed in map view (Figures 4 and 10) and a reasonable assumption can be made that infrasound should only originate from within the mapped region of movement. Individual arrays, whose signals evolve over time, can then be used to map candidate infrasound source locations as an intersection of this slide path and array back azimuth. We anticipate that Arrays A and C, which are situated with an oblique position relative to the avalanche path axis, have good capabilities for resolving translation of descending sources because the path comprises a large range of back azimuths. Conversely, stations such as Array B, which is located in the direction of the slide, have diminished resolving capabilities to track movement downslope. In some cases, changing apparent velocity (i.e., slowness amplitudes) may provide some constraint on source elevation (e.g., Marchetti et al., 2020), but elevation angles are not expected to vary much for our sensor distributions and so are not used to resolve locations in our analysis.

Infrasound source locations can be compared to visual advance of the snow avalanche extracted from video. Infrasound source regions are triangulated as the intersection of an array back azimuth and the slide path. In the Figure 11 maps, a grid search is used to estimate both the azimuths and timing of infrasound waves 


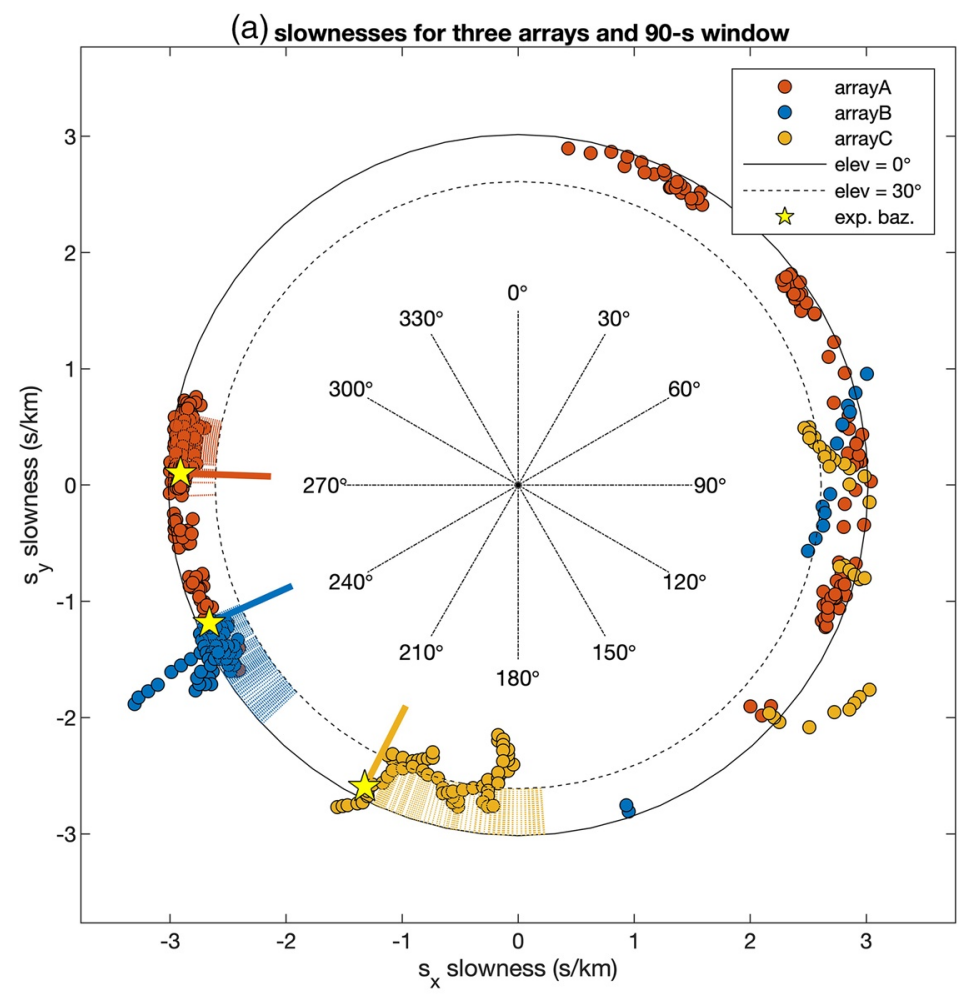

(b) backaziumths over time

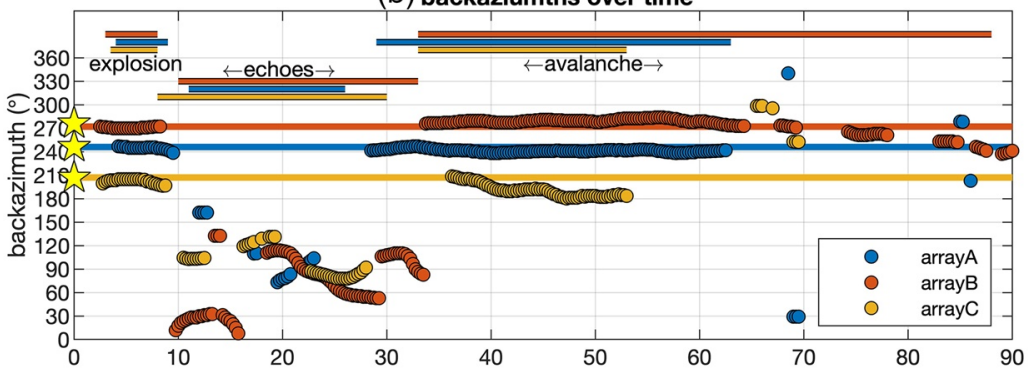

(c) infrasound intensity and video-derived

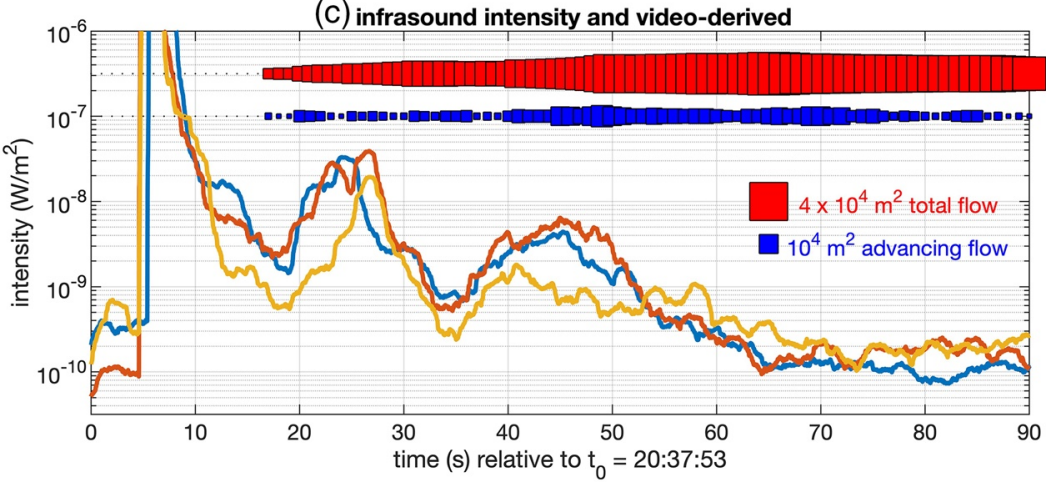

Figure 9. Back azimuth distribution and infrasound intensity chronology for the three arrays. (a) Back azimuth distribution is shown for a $90 \mathrm{~s}$ period of time with $5 \mathrm{~s}$ windows and $0.25 \mathrm{~s}$ time steps. Symbols located outside the solid circle, which corresponds to horizontally propagating infrasound, correspond to unreasonably high slownesses. Sources within the dashed and solid circles correspond to incident elevation angles between horizontal and $30^{\circ}$. Thin colored dashed lines correspond to the expected back azimuths for the active slide elements shown in Figure 2a. (b) Chronology of back azimuths for consistent, highly correlated infrasound energy is shown in time series. Echoes are identifiable as rapidly changing back azimuths for sound that is reflected back from crossvalley ridges and peaks. (c) The infrasound intensity time series showing three pulses corresponding to explosions, echoes, and avalanche infrasound is similar to Figure $6 \mathrm{~g}$. Records may be compared with video-derived avalanche motion whose symbol size (blue and red) denotes the time-varying area of total flow and advancing flow respectively. 


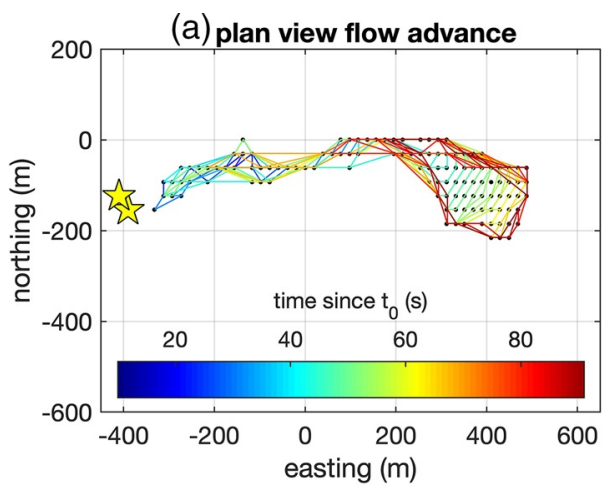

(b) profile view flow advance

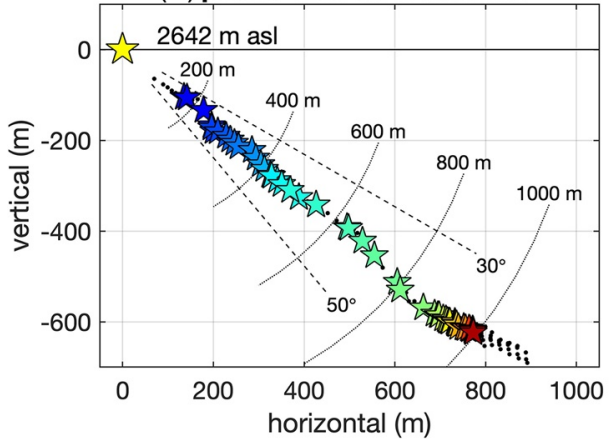

(c) flow position

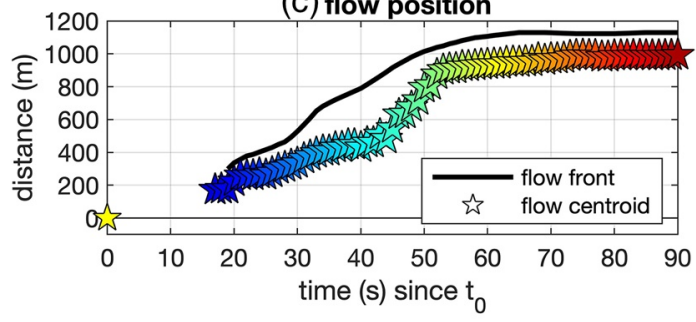

(d) flow velocity

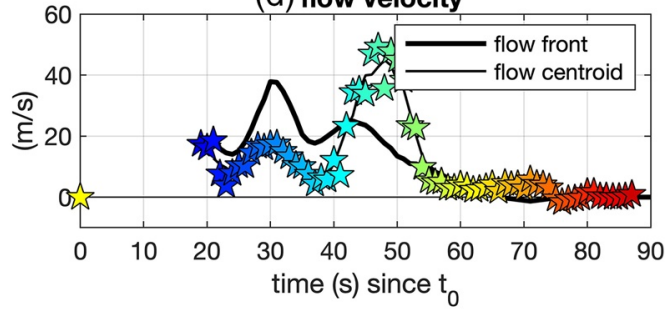

(e) flow acceleration

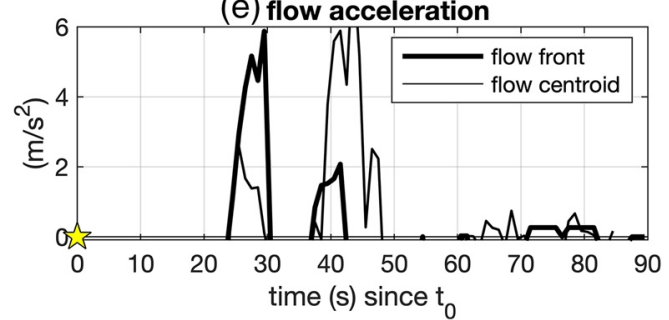



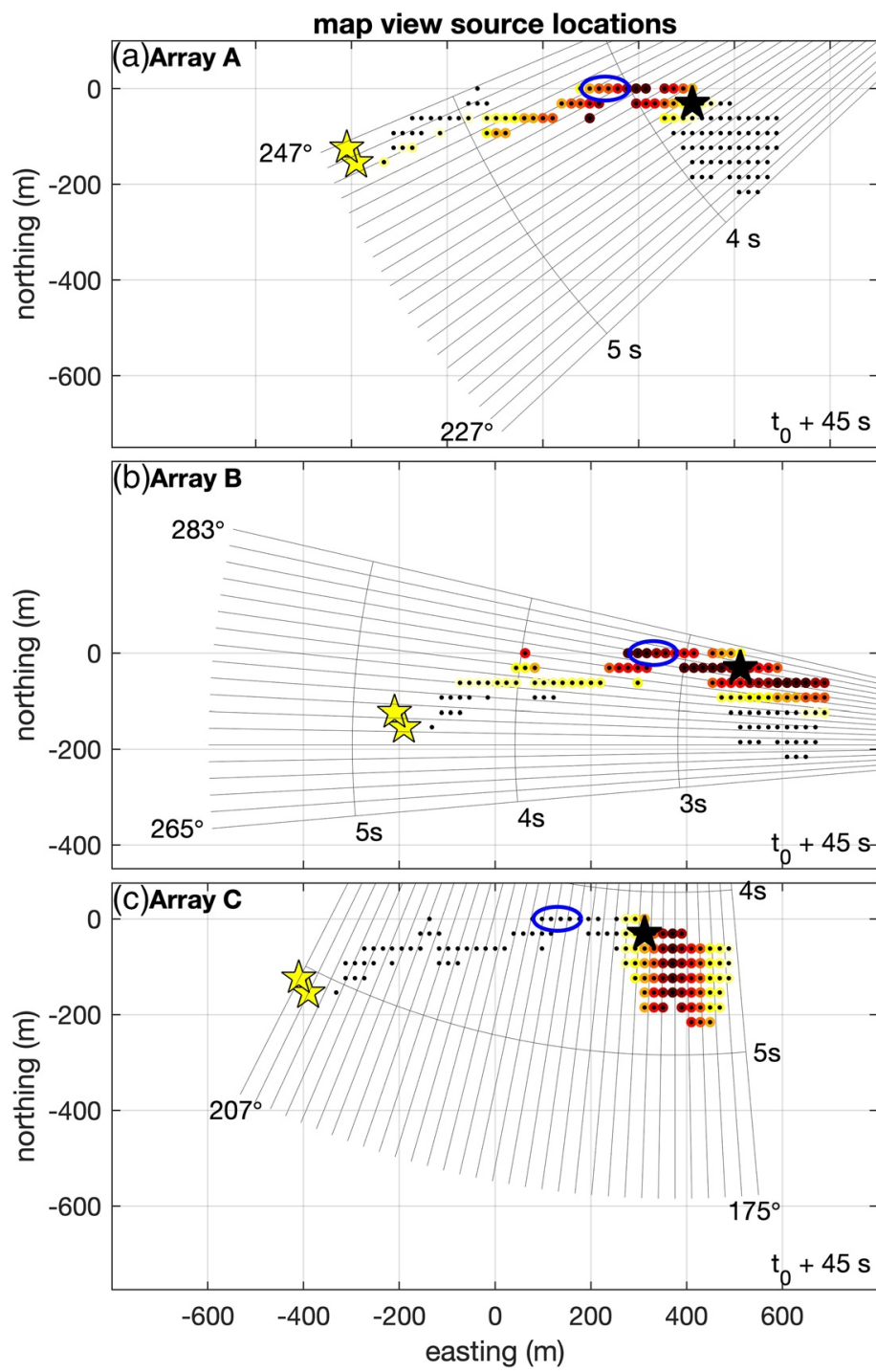
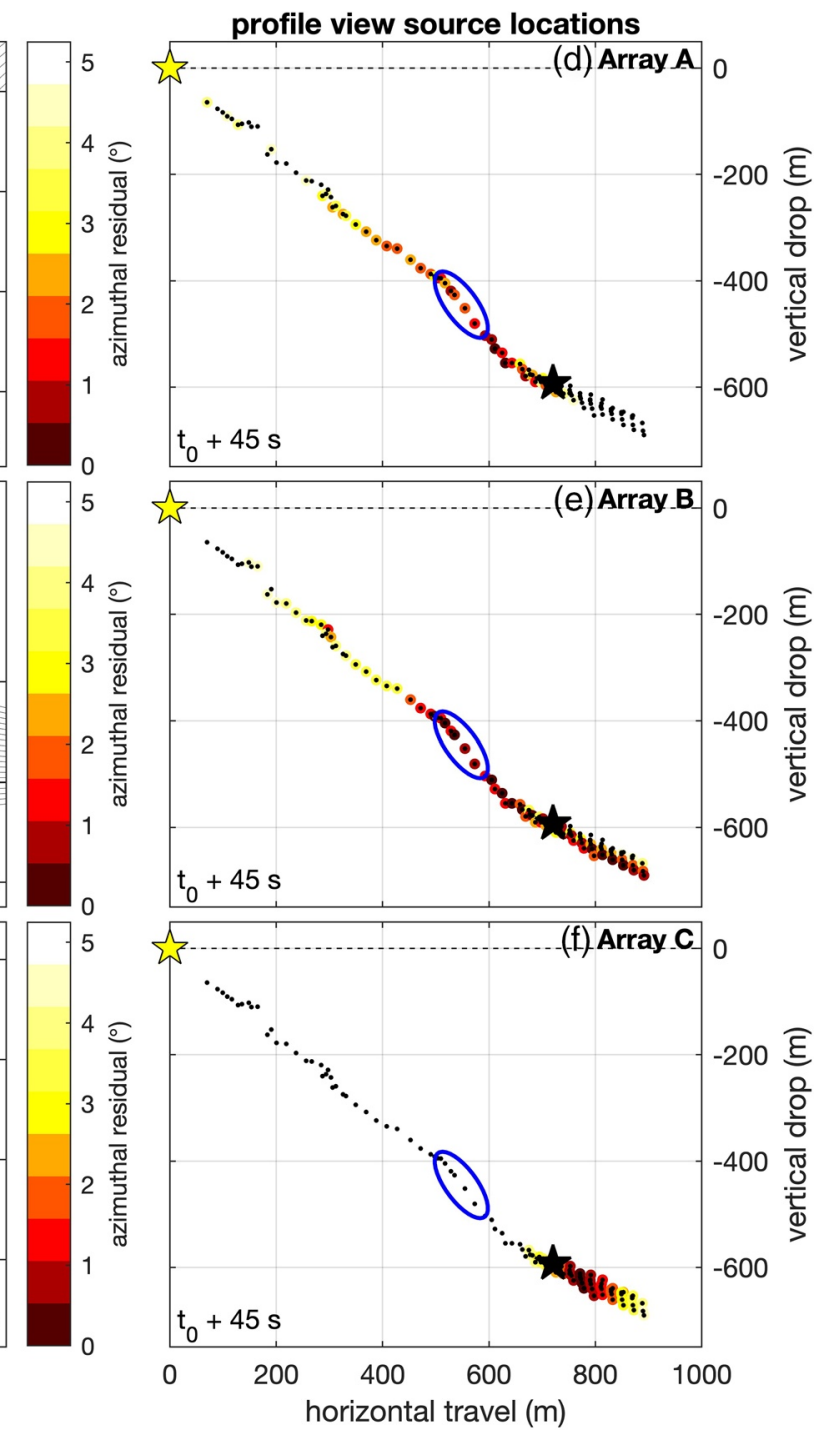

Figure 11. Array azimuthal residuals $\Theta$ and 3-array network infrasound residual minima are indicated for (a and d) Array A, (b and e) Array B, and (c and f) Array $\mathrm{C}$ for five second time windows centered at time $45 \mathrm{~s}$ after the explosion triggers. Black star corresponds to the minimum joint network residual $|\Theta|$. Blue ellipse corresponds to location of cliff band in both map and profile view. Azimuthal and propagation time contours relative to each array are indicated. A 70-s animated sequence of this figure is provided as supporting information (Movie S2).

reaching each array. Source-to-array residuals $\Theta$ are then defined as the difference between signal-derived infrasound azimuth $\theta_{s}$ and the calculated array azimuths $\theta_{c}$ for all candidate source positions $(x, y, z(x, y))$ and at each time step $(t)$, that is,

$$
\Theta=\left|\left(\theta_{s}(t)-\theta_{c}(x, y, t)\right)\right|
$$

The amplitudes of multiarray residuals (e.g., from the network of arrays A, B, and C) are then defined as

Figure 10. Chronology of Goat Slide avalanche extracted from video camera observations. (a) Active slide path regions shown in plan view where black dots indicate entire discretized source region and colored polygons represent active avalanche at successive $1 \mathrm{~s}$ time steps. (b) Profile view of the distance traveled showing mean location of the polygons from panel (a). Slant distance, relative to explosions, and slope angles are indicated. (c) Time/distance evolution of descending polygon centroids plotted with stars (using values from panel (b)) and the leading edge of the flow plotted as a solid black line. (d) Speeds are calculated as the time derivative of the distance chronology in panel (c) smoothed with a 5-s running window. (e) Flow acceleration is extracted from the time derivative of the velocity time series in panel (d). Yellow stars are explosion source locations as mapped in Figure 1. 


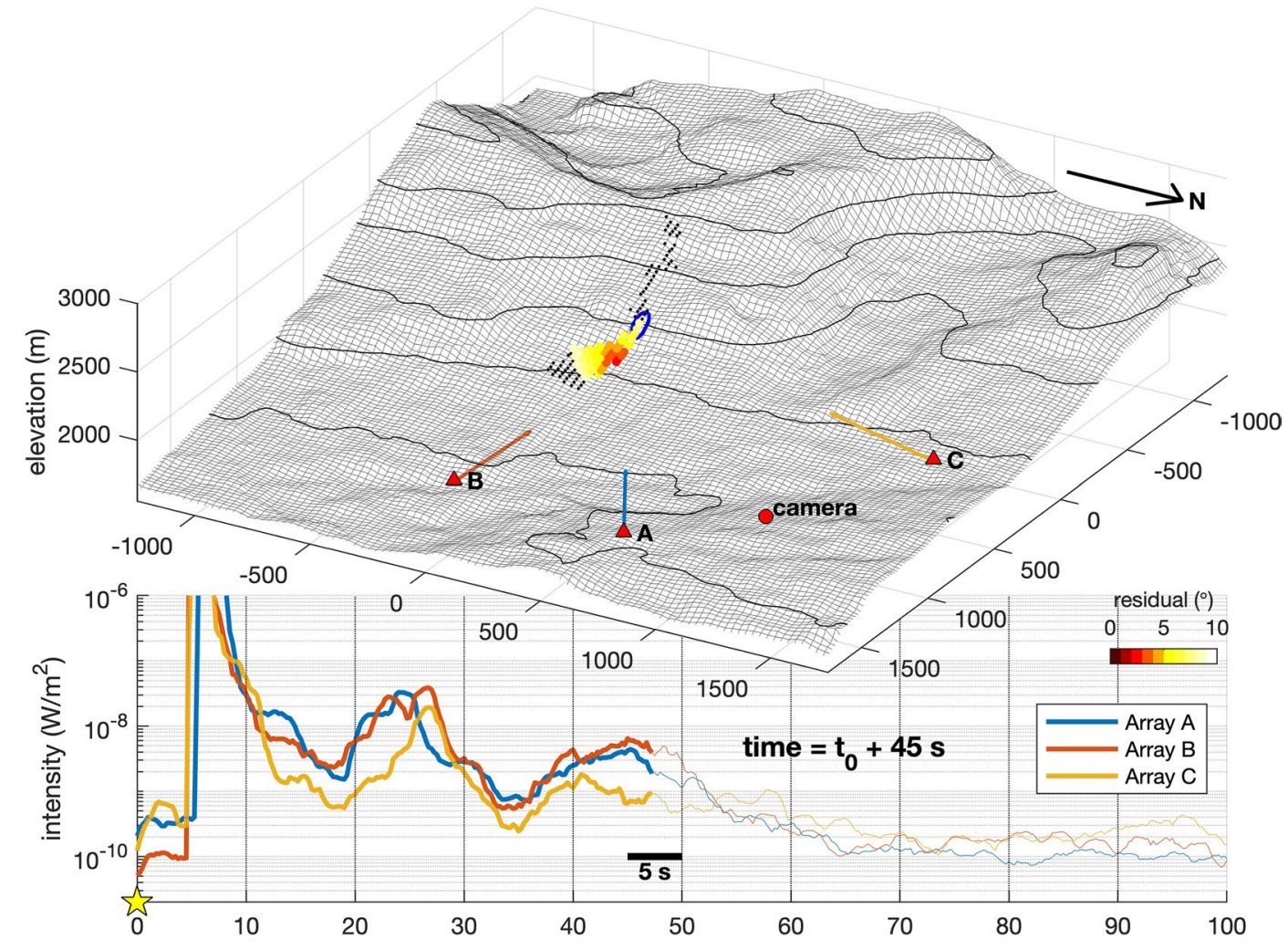

Figure 12. (Top) Infrasound source triangulation plotted as the intersection between slide path and the multistation residual $|\Theta|$ from three arrays in the network. Independent array back azimuths are indicated for each station for this time step centered at $t_{0}+45 \mathrm{~s}$. Blue ellipse corresponds to location of cliff band. (Bottom) Time-synchronized sound intensities, smoothed over $5 \mathrm{~s}$, and synchronized with mapped sources. Delay between explosion at $t_{0}$ and sound arriving at the various arrays is about $5 \mathrm{~s}$. Five-second analysis windows are indicated by black bar. A 70-s animated sequence of this figure is provided as supporting information (Movie S3). In the video, back azimuths are color-coded as black (for echoes), with long colored arrows (for explosions), and with short colored arrows (for slide path).

$$
|\Theta|=\sqrt{\sum_{j=A, B, C}\left(\theta_{j}-\theta_{j}(x, y, t)\right)^{2}}
$$

and can be used to estimate a primary locus of infrasound identified conjointly by all arrays.

Single arrays are only able to constrain the azimuthal direction to a source and, in the case where the avalanche path lies along a narrow range of azimuths (e.g., Array B), there is limited resolving capabilities for precision location of infrasound (see Figures $11 \mathrm{~b}$ and 11e). However, when an array is located oblique to the slide path, the source back azimuths sweep over a wider range of source positions permitting more precise localization of potential movement. Figures 11a, 11c, 11d, and 11f indicate that at $t=t_{0}+45 \mathrm{~s}$ the sources of infrasound appear to originate from near a change in slope and just below a topographic bottleneck and cliff band in the slide path. The lack of precise coincidence of the sources detected by Arrays A and C could be due to back azimuth uncertainties, or could be explained by the fact that the two different arrays are preferentially receiving infrasound from different parts of the flow. Given the distributed nature and large size of the flow path it is reasonable that the arrays have different sensitivities.

The direct comparison of infrasound source locations and avalanche infrasound time series is particularly illustrative. Synchronous display of infrasound power and mapped sources (Figure 12) reveal that the most intense infrasound from the Goat Slide event correspond to the advance of the flow over a mid-slidepath cliff band. Despite the visual movement of the slide lasting more than $60 \mathrm{~s}$ the primary infrasound 


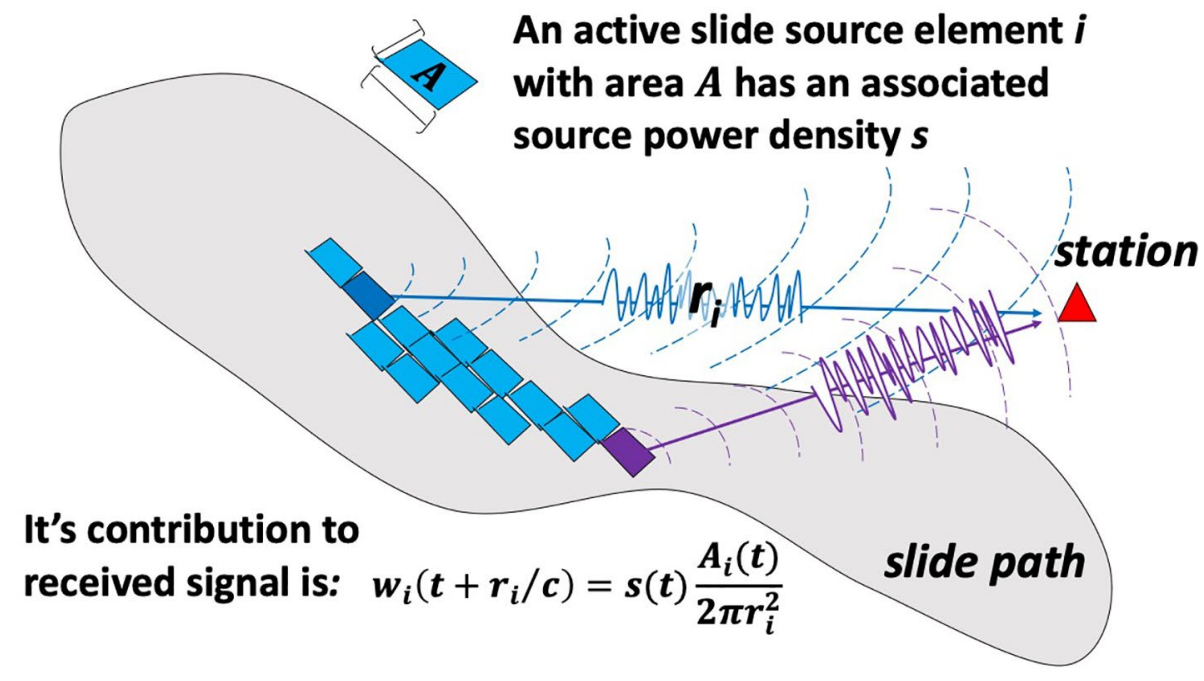

Figure 13. Schematic showing acoustic power contributions from discrete noncoherent source elements for a distribution of sources active at time step $\tau$.

production occurs only during a 20-s interval from 30 to $50 \mathrm{~s}$ after $t_{0}$ and this infrasound reaches the arrays from $t_{0}=35-55 \mathrm{~s}$.

\subsection{Infrasound Source Modeling}

We introduce here a methodology to compute acoustic source intensity for noncompact moving flow. We compare video-derived avalanche motion time series with infrasound intensity records from Arrays A, B, and $\mathrm{C}$ to derive an empirical relationship and understand which areas of the flow are most responsible for producing infrasound. Although the mapped Goat avalanche extent, using georegistered video, is subject to some spatial uncertainty, it is beneficial to analyze infrasound by incorporating these quantitative visual constraints.

Avalanches and debris flow are complex sources with noncompact source dimensions (e.g., Kogelnig et al., 2011; Marchetti et al., 2019). Here we treat the infrasound from the avalanche as a linear superposition of finite acoustic sources (Figure 13) distributed over a region $R$, which evolves over time. We propose a finite integration of infrasound sources, such as has been used for earthquakes (Arrowsmith et al., 2012) and volcanoes (Johnson \& Lees, 2010), to describe the infrasound generation by the noncompact avalanche. Because the avalanche infrasound is relatively structure-less in terms of its frequency spectrum across the near-infrasound $(2-25 \mathrm{~Hz}$ ) band, we model it here as a sum of incoherent sources distributed over the active portions of the slide path as seen in the video.

We estimate source acoustic intensity $s(\tau)$ by inversion of infrasound recordings considering video-based knowledge of the source's evolving geometry. The forward problem considers recorded infrasound intensity $I(t)$ to be a linear combination of signals from individual source elements, weighted and time shifted according to acoustic propagation. We assume that all source elements active at any given source time $\tau$ emit incoherent infrasound at a uniform power density $s(\tau)$.

We then divide the broad spatial area in which the avalanche occurs into potential source elements, and define $R(\tau)$ as the set of source elements determined by video analysis to be active at source time $\tau$. We consider and compare the two possible models articulated earlier in Figure 4c. These models of total flow (TF) and advancing flow (AF) correspond to the "entire spatial extent of moving snow" and the "change in spatial extent," respectively. We let $i \in R_{\tau}$ be a source element active at time $\tau$, with area $A_{i}$ and distance to receiver $r_{i}$ (Figure 13). Considering the travel time and hemispherical spreading between source and receiver, the contribution of this source element to the signal recorded at the receiver is 


$$
w_{i}\left(\tau+r_{i} / c\right)=s(\tau) \frac{A_{i}}{2 \pi r_{i}^{2}}
$$

which can be expressed using a transfer function incorporating a time shift

$$
\frac{A_{i}}{2 \pi r_{i}^{2}} \delta\left(t-\tau-r_{i} / c\right)
$$

where $\delta$ is the Dirac delta function. The collective transfer function of all source elements active at time $\tau$ is

$$
G_{\tau}(t)=\sum_{i \in R(\tau)}\left(\frac{A_{i}}{2 \pi r_{i}^{2}} \delta\left(t-\tau-r_{i} / c\right)\right)
$$

and the contribution of all source elements active at source time $\tau$ to the recorded infrasound is

$$
w_{\tau}(t)=G_{\tau}(t) s(\tau)
$$

When considering a superposition of source times, $G_{\tau}(t)$ can be written as a matrix where rows are source times $\tau$ and columns are recording times ( $t$ ) (Figures 14 and 15). The contributions of all source times and all weighted source elements is then

$$
I(t)=\sum_{\tau} G_{\tau}(t) s(\tau)
$$

Although this superposition is linear, the transfer function from source to receiver depends on the time-varying $R_{\tau}$ meaning that the system is not time-invariant and cannot be expressed as a convolution.

We can then invert for acoustic source intensity functions $S_{\tau}$ using the two different source models, total flow ( $I=G_{T F} S_{T F}$; Figure 14) and advancing flow $\left(I=G_{A F} S_{A F}\right.$; Figure 15). We solve using a nonnegative least squares inversion (Lawson \& Hanson, 1995), implemented in MATLAB with the function lsqnonneg.m, which optimizes the fit between modeled and observed infrasound power while avoiding unphysical negative source power. The strategy and reasoning are similar to the technique outlined in Anderson et al. (2014) for locating acoustic source strengths associated with thunder, whose lightning sources are also extensive in area, but_unlike avalanches_-are instantaneous in time. Solutions for infrasound intensity are computed separately for the data from all three arrays $\left(I_{A}, I_{B}\right.$, and $\left.I_{C}\right)$ and show relatively consistent source intensities (to within a factor of about 3 ) for each solution $\left(S_{A}, S_{B}\right.$, and $S_{C}$; Figures $14 \mathrm{c}$ and $15 \mathrm{c}$ ). When the source strength is computed using only the advancing portion of the flow its spatial extent is smaller and, as intuition suggests, the corresponding modeled source intensity is greater than for the total flow model.

We consider the solutions for total flow and advancing flow to be end-member infrasound source intensities for this avalanche. Integrated over the $\sim 20 \mathrm{~Hz}$ band (2-25 Hz and excluding notches) the calculated peak source intensity at around $t_{0}+40 \mathrm{~s}$ ranges from $S_{T F}=0.4 \pm 0.2 \mu \mathrm{W} / \mathrm{m}^{2}$ to $S_{A F}=1.8 \pm 0.7 \mu \mathrm{W} / \mathrm{m}^{2}$. At the receivers, these peak intensities occur at around $t_{0}+45 \mathrm{~s}$ and have values ranging from $0.02 \mu \mathrm{W} / \mathrm{m}^{2}$ (Array C) to $0.06 \mu \mathrm{W} / \mathrm{m}^{2}$ (for Arrays A and B).

\section{Discussion}

\subsection{Interpretation of Source Inversions and Relationship With Video Observations}

The infrasound source models depicted in Figures $14 \mathrm{c}$ and $15 \mathrm{c}$ have a distinct high-amplitude pulse on all three stations occurring from 30 to $50 \mathrm{~s}$ after $t_{0}$. Significantly, this appears related to flow acceleration and passage through the mid-path cliff band (Figure 16). In all six cases (three arrays and two modeled source types), the maximum source intensity is relatively short in duration compared to the $\sim 60 \mathrm{~s}$ visual manifestation of the Goat event, which is evident in the video from around 15 to $75 \mathrm{~s}$ after $t_{0}$. In all six cases, the infrasound source functions reach maximum value at around $t_{0}+39 \pm 3 \mathrm{~s}$ corresponding to the time when the avalanche begins to spill over the cliff band. This peak infrasound source power does not correlate well 


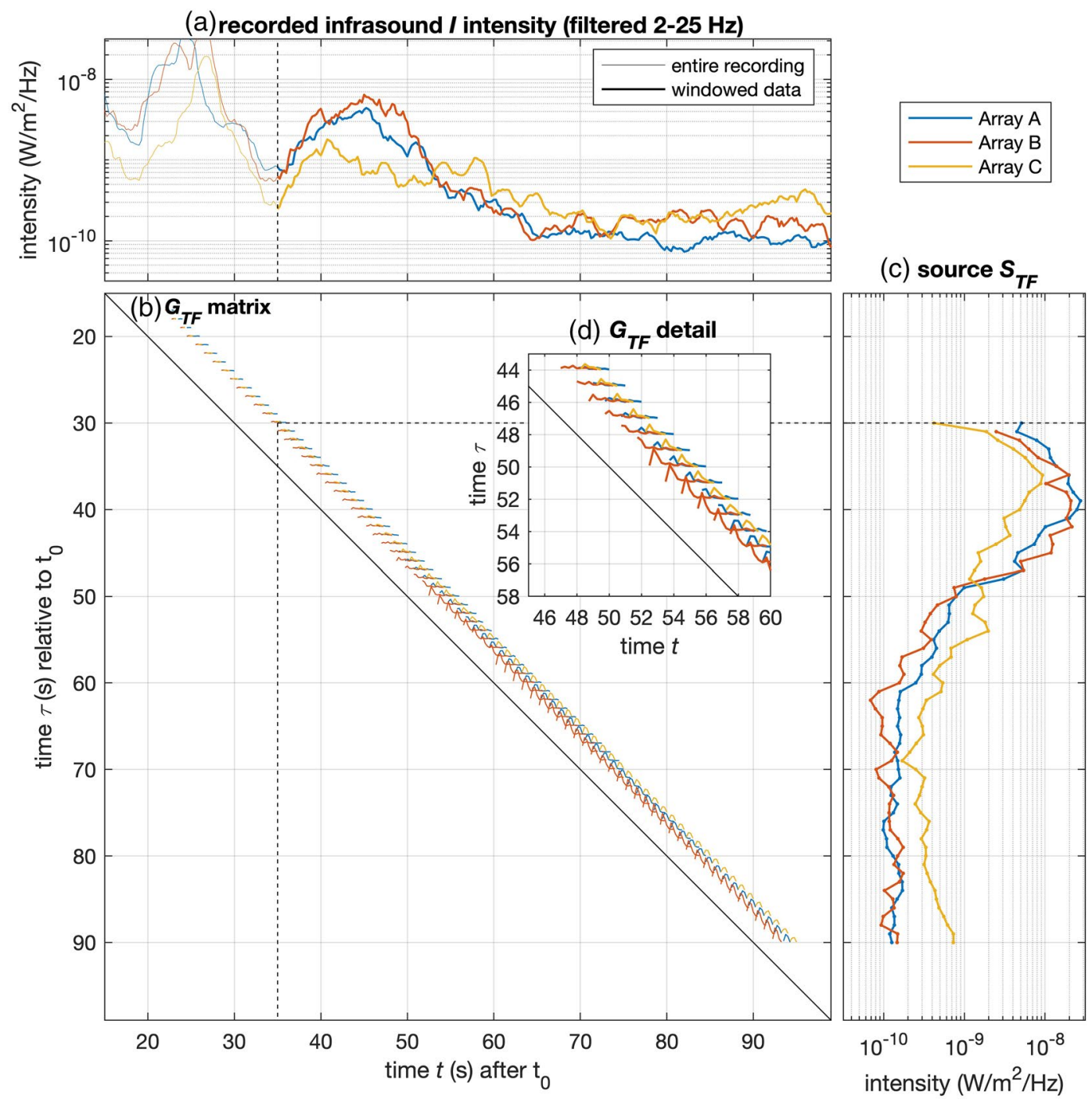

Figure 14. Schematic calculation of the time-varying acoustic source intensity for total flow (TF) model. (a) Recorded acoustic intensities $(I)$ are indicated for three arrays and with logarithmic scale. (b) Graphical representation of source convolution functions for each $1 \mathrm{~s}$ time step using the total flow distribution (blue symbols in Figure 4c). Multipliers in panel (c) correspond to source intensity, which is computed for the windowed data time period after the echoes have subsided. (d) Inset provides example detail of convolution functions comprising G.

with video-derived flow velocity maxima, but instead appears to be more consistent with the avalanche's peak acceleration (Figure 16).

We suggest that flow acceleration may be an important predictor of the intensity of the infrasound produced, that is, fast-accelerating flows may be particularly well suited to generating intense infrasound. The fastest accelerating portions of the flow cascading over the cliff band appear to produce the most intense infrasound, while the trailing edge of the avalanche (e.g., after $t_{0}+50 \mathrm{~s}$ ), in which the powder cloud traverses a relative flat runout zone, is notably quiet. This trailing portion of the avalanche is still visually impressive in terms of its large, white cloud, yet it does not manifest as infrasonically "loud."

Generation of infrasound has previously been attributed with the production, or presence, of a powder cloud (e.g., Marchetti et al., 2020), but our analysis indicates a preponderance of infrasound produced only during the initial generation of the powder cloud as the flow moves over a steep drop. As the flow accelerates over the mid-path cliff band, the suspension layer of the flow is likely to behave turbulently, which has been proposed as the infrasound generation mechanism in Kogelnig et al. (2011). We suggest that the 


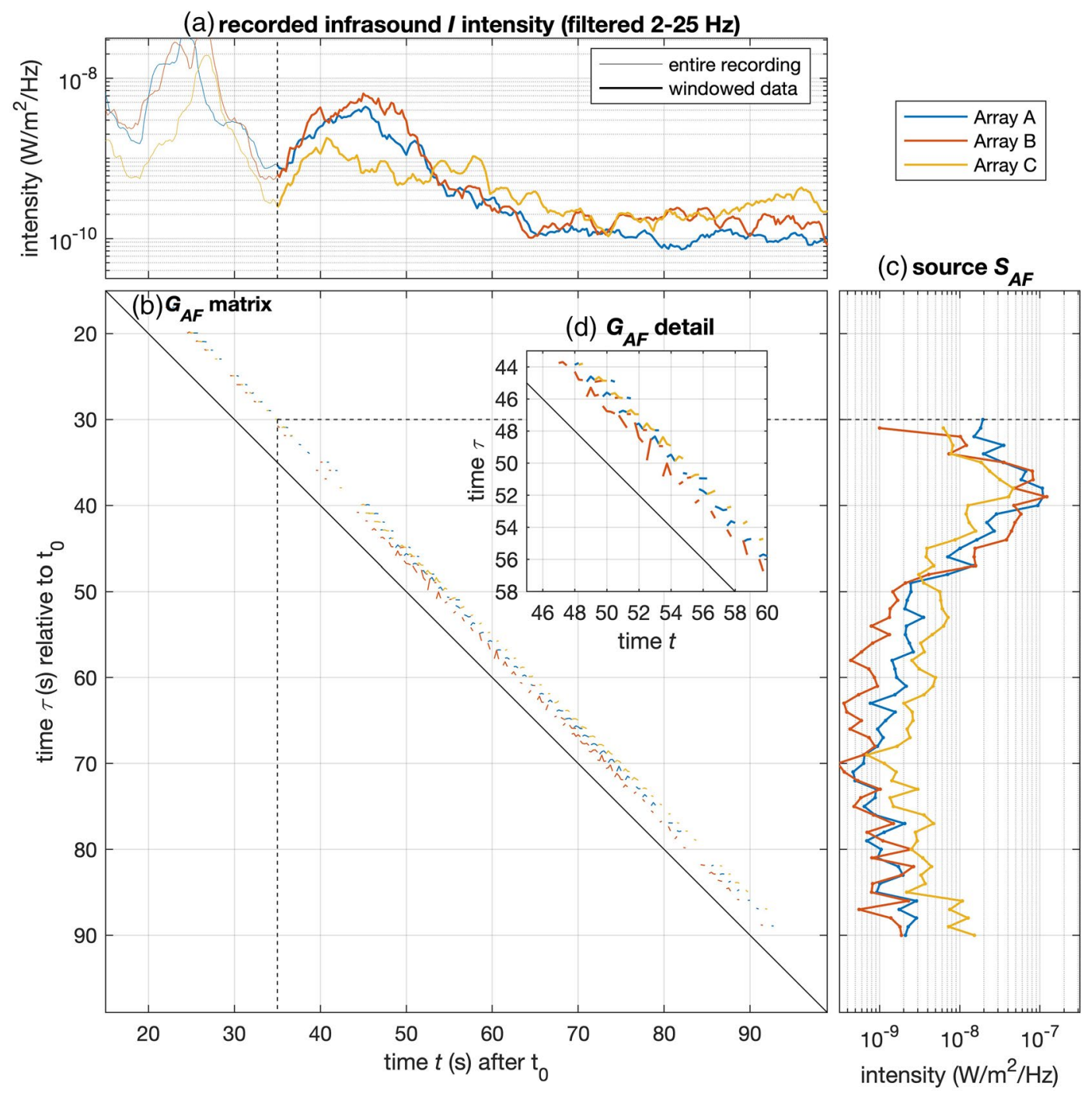

Figure 15. Calculation of acoustic source intensity for advancing flow model showing (a) recorded acoustic intensities, (b) source convolution functions, (c) source intensities, and (d) detail of convolution functions. Caption is same as for Figure 14, but $\mathrm{G}$ matrix is computed using a finite source distribution limited to the advancing flow (AF; red symbols in Figure $4 \mathrm{c})$.

advancing powder cloud after $t_{0}+50 \mathrm{~s}$ either does not have a sufficient density contrast to significantly push on the overlying atmosphere and/or that its turbulent motion is diminished relative to the early, faster accelerating portion of the flow. We note that our observation also contrasts with that of Havens et al. (2014), who correlated the most intense portion of their infrasonic record to the arrival of an avalanche at the valley floor. In their case, the valley floor represented an abrupt transition from steep slopes where avalanche deceleration was likely more dramatic.

\subsection{Limitations of Source Intensity Inversions}

The inverted source acoustic models are moderately different when using data from different arrays, suggesting that the current modeling is relatively simplistic. We suggest that several improvements might be made in future work to more accurately invert for source parameters. First, discretization of the source geometry may be improved with better digital elevation models, more suitable camera viewing geometries, and cameras with better contrast and/or IR sensitivity. Second, geometric spreading losses may be better modeled through inclusion of topography; given that portions of the flow path are not line-of-sight to all 

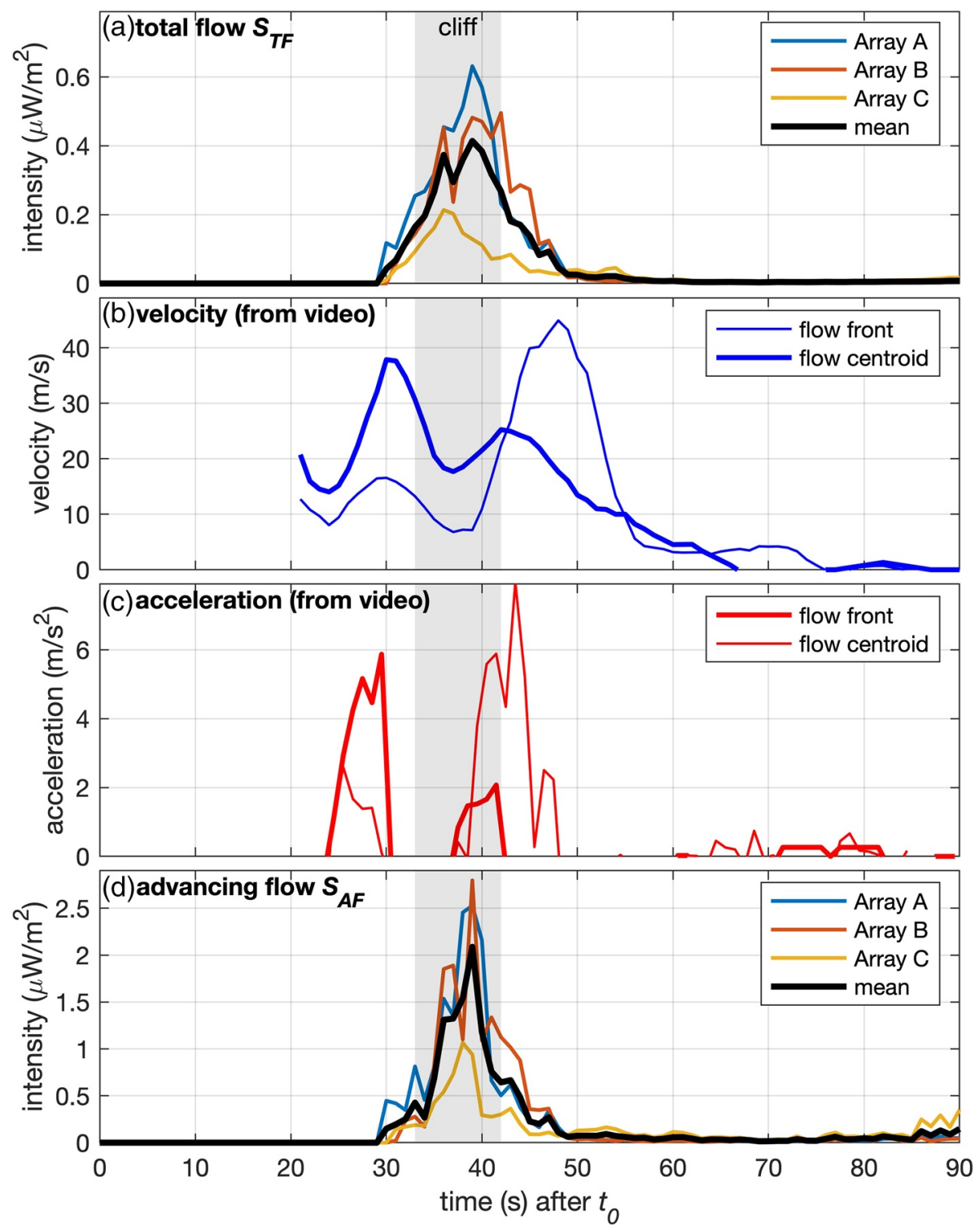

Figure 16. Relationship between modeled infrasound source intensity and flow advance parameters extracted from video (refer to Figure 10). (a) Time series for total flow source intensity. (b) Velocity time series for the flow centroid and leading flow edge. (c) Acceleration time series for the flow centroid and leading flow edge. (d) Time series for advancing flow source intensity. Gray shading indicates the time when the flow spills over the steep cliff band as seen in video records (Movie S1).

three arrays at all times, we suspect that diffraction as well as some intrinsic attenuation of the propagating infrasound is occurring. Third, that the recorded avalanche infrasound may be partially obscured by echoes and helicopters means that we are not characterizing the full spectral and temporal extent of the avalanche infrasound. Studies without these sources of noise would be preferable. Lastly, and perhaps most significantly, our inversion treats each element of the flow path as being uniformly strong for a given time step and as being spatially noncoherent (e.g., white noise). As such, intensity calculated from TF and AF distributions represent a reasonable range of source intensities that could be more precisely calculated in future studies. Additional constraints, including observations from Doppler radars (e.g., Köhler et al., 2016; Marchetti et al., 2015) would be helpful for pinpointing the timing of flow surges and which portions of the flow are most energetic. 


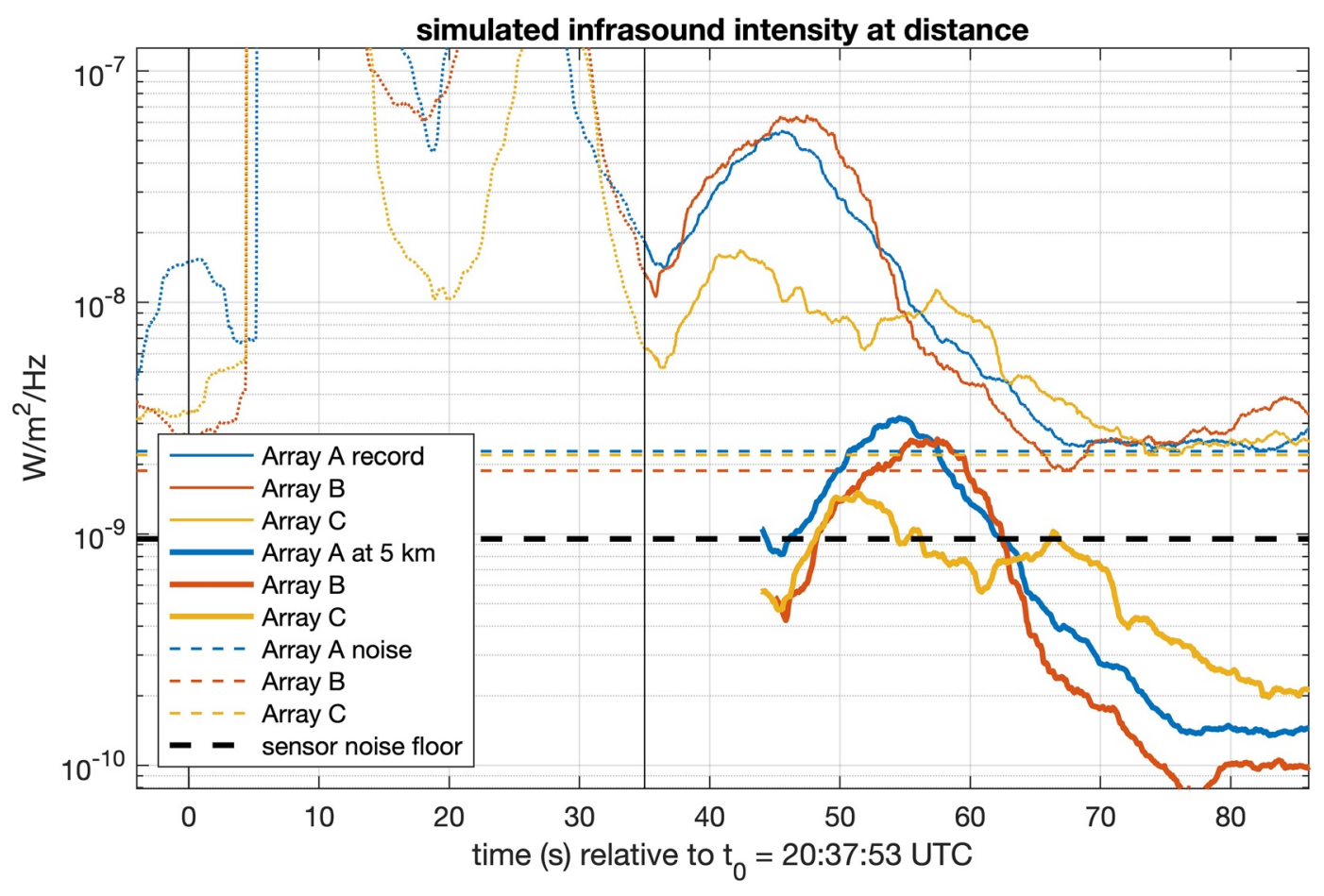

Figure 17. Recorded infrasound intensity time series derived from Figure 6g, but with estimated amplitudes reduced to a common recording distance of $5 \mathrm{~km}$ (assuming avalanche signal is primarily sourced from the mid-slide cliff band). A time shift has also been applied to estimate propagation time out to $5 \mathrm{~km}$. The noise floors are taken as the rootmean-squared minimum values recorded at stations A, B, and C during the 90-s event window. The indicated sensor noise floor corresponds to the $1-10 \mathrm{~Hz}$ RMS noise of the infraBSU_vers01 sensor.

\subsection{Estimation of Maximum Distance for Avalanche Detectability}

The Goat Slide event was a relatively small, dry avalanche that produced relatively low-intensity infrasound; however, its study can lead to an estimate of ideal deployment topologies for detecting and studying events of comparable size. The band-limited intensity plots introduced in Figure 6 give an indication of detection capabilities for arrays that could be located farther away. Given that the source-to-receiver distances are less than $2 \mathrm{~km}$ it is possible to extrapolate to further distances to estimate where infrasound might no longer be detectable.

Consideration of sensor noise floors, as well as the level of ambient noise in the recording environment, is critical for this exercise. For the recordings made at Arrays A, B, and C (which are about 1.2, 1.0, and $1.5 \mathrm{~km}$ from the cliff-band in the Goat Path), we can predict what the infrasound intensities might be at further distances. At a distance of about $5 \mathrm{~km}$ from the cliff, for instance, the intensity would be diminished primarily by geometric spreading, but probably not impacted significantly by intrinsic attenuation (Kogelnig et al., 2014). Assuming a compact source of infrasound produced at the cliff band and spreading hemispherically, acoustic intensity would decrease with the square of distance, such that estimated levels at $5 \mathrm{~km}$ would be reduced by 10,9 and $12 \mathrm{~dB}$ at arrays A, B, and C respectively (Figure 17). We suggest that these infrasound levels would be barely perceptible above the ambient noise $\left(\sim 2 \times 10^{-9} \mathrm{~W} / \mathrm{m}^{2} /\right.$ $\mathrm{Hz}$ ) that was present during the campaign of March 13, 2018. This ambient noise was only slightly higher than the theoretical instrument self-noise of the infrasound sensors $\left(1 \times 10^{-9} \mathrm{~W} / \mathrm{m}^{2} / \mathrm{Hz}\right)$ meaning that in ideal recording conditions (still air) the Goat event that was triggered at 20:37:53 UTC would probably not have been detected beyond about $5 \mathrm{~km}$. Understanding the distance limits on detection capabilities for avalanches as a function of size is an important goal of avalanche forecasters and practitioners (e.g., Mayer et al., 2020). 


\subsection{Future Research Direction}

Understanding the relationship between infrasound production and flow energetics will benefit from additional field experiments, involving multidisciplinary techniques such as radars and photogrammetric methods. Focused infrasound study incorporating these integrated observations, such as is routinely done at the Vallee de la Sionne (e.g., Köhler et al., 2016; Pérez-Guillén et al., 2014), will provide vital validation for flow parameters that cannot be directly measured with infrasound remote sensing. Coupled infrasound and seismic wave analysis, which has been used on its own to quantify flow parameters (e.g., Lacroix et al., 2012; Marchetti et al., 2020; Van Herwijnen \& Schweizer, 2011; Vilajosana et al., 2007), will be especially useful for understanding behaviors of avalanches of variable size, variable density, (e.g., wet vs. dry) and for different types of flow regimes including basal flow, fluidized, and suspension layers (Gauer et al., 2008).

An improved understanding and modeling of the infrasound produced by snow avalanches of various types might then be applied to other gravity-driven mass movements including rock fall, debris flows, or pyroclastic density currents at volcanoes. Given the common occurrence of snow avalanches and relatively easy logistics involved in their observation, it makes sense to use snow avalanches as a test bed for understanding the physics and sound-generation mechanisms of other types of rapid, and potentially hazardous, mass wasting.

\section{Conclusion}

Infrasound has been used for decades to detect the occurrence of snow avalanches, but only recently has it been developed as a tool to quantify more detailed flow parameters including velocities and source energetics. We have shown that coupled visual and infrasound observations can be used together to provide estimates of the acoustic source intensity of advancing flows. An accurate understanding of avalanche sound source power is important, for instance, because it might be used to estimate infrasound detection thresholds for flows of various size and source-receiver distances.

Our study combining visual and infrasound observations from a network of arrays is used to identify a midflow cliff band as the region of most significant infrasound production and to quantify the source acoustic power density over time. These results are a step forward toward developing more sophisticated models that may predict the infrasound radiated by a range of snow avalanches types (e.g., small, large, wet, dry). Because infrasound is recognized as an efficient, cost-effective technology to monitor snow avalanches, and to potentially minimize their hazard impact, an improved understanding of infrasound generation physics is beneficial. Integrated visual-infrasound studies are of importance to both researchers and practitioners who wish to understand infrasound detection efficiency and map avalanches as accurately as possible.

Acknowledgments

We thank the Transportation Avalanche Research Pool (TARP) for their support of snow avalanche infrasound research through Grant 3411019021 awarded to Boise State University (BSU). Partial support for this work is also credited to BSU grant \#W913E520C0017 from the U.S. Cold Regions Research and Engineering Laboratory (CRREL) Broad Agency Announcement (BAA) and to BSU award \#1914491 from the National Science Foundation (NSF) Division of Earth Science (EAR). We acknowledge with appreciation The British Broadcasting Company (BBC), and Caravan Media producer Ian White, who supported us and organized access and logistics to conduct this experiment in Alberta. Engaging discussion with researchers Jim McElwaine and Chris Hopkinson, and Utah Department of Transportation (UDOT) avalanche forecaster Mark Saurer served to improve this manuscript.

\section{Data Availability Statement}

Infrasound waveform data analyzed in this study are available for public download from a BSU ScholarWorks archive focused on infrasound datasets accessible at https://scholarworks.boisestate.edu/infrasound_data/. These data are archived with the dedicated searchable digital object identifier 10.18122/infrasound_data/4/boisestate. A digital elevation model was obtained from the NASA Shuttle Radar Topography Mission (SRTM) Version 3.0 Global arc-second resource.

\section{References}

Adam, V., Chritin, V., Rossi, M., \& van Lancker, E. (1998). Infrasonic monitoring of snow-avalanche activity. What do we know and where do we go from here? Annals of Glaciology, 26, 324-328.

Anderson, J. F., Johnson, J. B., Arechiga, R. O., \& Thomas, R. J. (2014). Mapping thunder sources by inverting acoustic and electromagnetic observations. Journal of Geophysical Research: Atmospheres, 119(23), 13287-13304. https://doi.org/10.1002/2014JD021624

Arai, N., Imai, T., Otsuki, M., Saito, Y., Murayama, T., \& Iwakuni, M. (2017). Detection of avalanche locations using infrasound array data. Bulletin of Glaciological Research, 35, 1-6. https://doi.org/10.5331/bgr.16R02

Arrowsmith, S. J., Burlacu, R., Pankow, K., Stump, B., Stead, R., Whitaker, R., \& Hayward, C. (2012). A seismoacoustic study of the 2011 January 3 Circleville earthquake. Geophysical Journal International, 189(2), 1148-1158. https://doi.org/10.1111/j.1365-246X.2012.05420.x

Bedard, A. J., Greene, G. E., Intrieri, J., \& Rodriguez, R. (1988). On the feasibility and value of detecting and characterizing avalanches remotely by monitoring radiated sub-audible atmospheric sound at long distances (pp. 267-275). Proceedings of A Multidisciplinary approach to Snow Engineering, U.S. Army Corps of Engineers; Cold Regions Research and Engineering Laboratory, Santa Barbara, CA. 
Cansi, Y. (1995). An automatic seismic event processing for detection and location: The P.M.C.C. Method. Geophysical Research Letters, 22(9), 1021-1024. https://doi.org/10.1029/95GL00468

Comey, R., \& Mendenhall, T. (2004). Recent studies using infrasound sensors to remotely monitor avalanche activity (pp. 19640-24646). Proceeding of International Snow Science Workshop, WY, Jackson.

Delle Donne, D., Ripepe, M., De Angelis, S., Cole, P. D., Lacanna, G., Poggi, P., \& Stewart, R. (2014). Chapter 9 thermal, acoustic and seismic signals from pyroclastic density currents and Vulcanian explosions at Soufrière Hills Volcano, Montserrat. Geological Society, London, Memoirs, 39(1), 169-178. https://doi.org/10.1144/M39.9

Gauer, P., Issler, D., Lied, K., Kristensen, K., \& Sandersen, F. (2008). On snow avalanche flow regimes: Inferences from observations and measurements. Proceedings of the International Snow Science Workshop ISSW '08, Whistler, BC, Canada.

Greene, E., Birkeland, K. W., Elder, K., Mccammon, I., Staples, M., \& Sharaf, D. (2016). Snow, weather and avalanches: Observation Guidelines for Avalanche Programs in the United States (SWAG). American Avalanche Association. Retrieved from http://www.americanavalancheassociation.org/swag/

Havens, S., Marshall, H.-P., Johnson, J. B., \& Nicholson, B. (2014). Calculating the velocity of a fast-moving snow avalanche using an infrasound array. Geophysical Research Letters, 41(17), 6191-6198. https://doi.org/10.1002/2014GL061254

Heck, M., Hobiger, M., van Herwijnen, A., Schweizer, J., \& Fäh, D. (2019). Localization of seismic events produced by avalanches using multiple signal classification. Geophysical Journal International, 216(1), 201-217. https://doi.org/10.1093/gji/ggy394

Johnson, J. B., \& Lees, J. M. (2010). Sound produced by the rapidly inflating Santiaguito lava dome, Guatemala. Geophysical Research Letters, 37(22), L22305. https://doi.org/10.1029/2010GL045217

Johnson, J. B., Marshall, H. P., Loo, S. M., Nalli, B., Saurer, M., Havens, S., et al. (2018). Detection and tracking of snow avalanches in Little Cottonwood Canyon, Utah using multiple small-aperture infrasound arrays. Proceeding of International Snow Science Workshop, Innsbruck, Austria, 7-12 October 2018.

Johnson, J. B., \& Palma, J. L. (2015). Lahar infrasound associated with Volcán Villarrica’s 3 March 2015 eruption. Geophysical Research Letters, 42(15), 6324-6331. https://doi.org/10.1002/2015GL065024

Johnson, J. B., \& Ronan, T. J. (2015). Infrasound from volcanic rockfalls. Journal of Geophysical Research: Solid Earth, 120(12), 8223-8239. https://doi.org/10.1002/2015JB012436

Kogelnig, A., Hübl, J., Suriñach, E., Vilajosana, I., \& McArdell, B. W. (2014). Infrasound produced by debris flow: Propagation and frequency content evolution. Natural Hazards, 70(3), 1713-1733. https://doi.org/10.1007/s11069-011-9741-8

Kogelnig, A., Suriñach, E., Vilajosana, I., Hübl, J., Sovilla, B., Hiller, M., \& Dufour, F. (2011). On the complementariness of infrasound and seismic sensors for monitoring snow avalanches. Natural Hazards and Earth System Sciences, 11(8), 2355-2370. https://doi.org/10.5194/ nhess-11-2355-2011

Köhler, A., McElwaine, J. N., Sovilla, B., Ash, M., \& Brennan, P. (2016). The dynamics of surges in the 3 February 2015 avalanches in Vallée de la Sionne. Journal of Geophysical Research: Earth Surface, 121(11), 2192-2210. https://doi.org/10.1002/2016JF003887

Lacroix, P., Grasso, J. R., Roulle, J., Giraud, G., Goetz, D., Morin, S., \& Helmstetter, A. (2012). Monitoring of snow avalanches using a seismic array: Location, speed estimation, and relationships to meteorological variables. Journal of Geophysical Research: Earth Surface, 117(1), 1-15. https://doi.org/10.1029/2011JF002106

Lawson, C. L., \& Hanson, R. J. (1995). Solving least squares problems (NV-1 onl). Philadelphia, PA: Society for Industrial and Applied Mathematics. Retrieved from http://epubs.siam.org/ebooks/siam/classics_in_applied_mathematics/cl15

Liu, D., Leng, X., Wei, F., Zhang, S., \& Hong, Y. (2015). Monitoring and recognition of debris flow infrasonic signals. Journal of Mountain Science, 12(4), 797-815. https://doi.org/10.1007/s11629-015-3471-4

Marchetti, E., Ripepe, M., Ulivieri, G., \& Kogelnig, A. (2015). Infrasound array criteria for automatic detection and front velocity estimation of snow avalanches: Towards a real-time early-warning system. Natural Hazards and Earth System Sciences Discussions, 3(4), 2709-2737. https://doi.org/10.5194/nhessd-3-2709-2015

Marchetti, E., Van Herwijnen, A., Christen, M., Cristina Silengo, M., \& Barfucci, G. (2020). Seismo-acoustic energy partitioning of a powder snow avalanche. Earth Surface Dynamics, 8(2), 399-411. https://doi.org/10.5194/esurf-8-399-2020

Marchetti, E., Walter, F., Barfucci, G., Genco, R., Wenner, M., Ripepe, M., et al. (2019). Infrasound array analysis of debris flow activity and implication for early warning. Journal of Geophysical Research: Earth Surface, 124(2), 567-587. https://doi.org/10.1029/2018JF004785

Marcillo, O., Johnson, J. B., \& Hart, D. (2012). Implementation, characterization, and evaluation of an inexpensive low-power low-noise infrasound sensor based on a micromachined differential pressure transducer and a mechanical filter. Journal of Atmospheric and Oceanic Technology, 29(9), 1275-1284. https://doi.org/10.1175/JTECH-D-11-00101.1

Mayer, S., van Herwijnen, A., Ulivieri, G., \& Schweizer, J. (2020). Evaluating the performance of an operational infrasound avalanche detection system at three locations in the Swiss Alps during two winter seasons. Cold Regions Science and Technology, 173, 102962. https:// doi.org/10.1016/j.coldregions.2019.102962

Pérez-Guillén, C., Tapia, M., Furdada, G., Suriñach, E., McElwaine, J. N., Steinkogler, W., \& Hiller, M. (2014). Evaluation of a snow avalanche possibly triggered by a local earthquake at Vallée de la Sionne, Switzerland. Cold Regions Science and Technology, 108, 149-162. https://doi.org/10.1016/j.coldregions.2014.07.007

Preiswerk, L., Walter, F., Anandakrishnan, S., Barfucci, G., Beutel, J., Burkett, P., et al. (2016). Monitoring unstable parts in the ice-covered Weissmies northwest face (pp. 434-443). Proceedings of the 13th Interpraevent Congress, Lucerne. https://doi.org/10.3929/ ethz-a-010811690

Richardson, J. P., Waite, G. P., FitzGerald, K. A., \& Pennington, W. D. (2010). Characteristics of seismic and acoustic signals produced by calving, Bering Glacier, Alaska. Geophysical Research Letters, 37(3). https://doi.org/10.1029/2009GL041113

Scott, E. D., Hayward, C. T., Kubichek, R. F., Hamann, J. C., Pierre, J. W., Comey, B., \& Mendenhall, T. (2007). Single and multiple sensor identification of avalanche-generated infrasound. Cold Regions Science and Technology, 47, 159-170. https://doi.org/10.1016/j. coldregions.2006.08.005

Stubbs, C., Brenner, M., Flatt, S., Goodman, J., \& Hearing, B. (2005). Tactical infrasound. McLean, Virginia: Mitre Corp Mclean Va Jason Program Office.

Ulivieri, G., Marchetti, E., Ripepe, M., Chiambretti, I., De Rosa, G., \& Segor, V. (2011). Monitoring snow avalanches in Northwestern Italian Alps using an infrasound array. Cold Regions Science and Technology, 69(2-3), 177-183. https://doi.org/10.1016/j.coldregions.2011.09.006

Van Herwijnen, A., \& Schweizer, J. (2011). Seismic sensor array for monitoring an avalanche start zone: Design, deployment and preliminary results. Journal of Glaciology, 57(202), 267-276. https://doi.org/10.3189/002214311796405933

Vilajosana, I., Khazaradze, G., Suriñach, E., Lied, E., \& Kristensen, K. (2007). Snow avalanche speed determination using seismic methods. Cold Regions Science and Technology, 49(1), 2-10. https://doi.org/10.1016/j.coldregions.2006.09.007 
Yount, J., Naisbitt, A., Scott, E. D., \& Laboratories, I. (2008). Operational highway avalanche forecasting using the infrasonic avalanche (pp. 268-276). International Snow Science Workshop Proceedings, Whistler, BC.

Zimmer, V. L., Collins, B. D., Stock, G. M., \& Sitar, N. (2012). Rock fall dynamics and deposition: An integrated analysis of the 2009 Ahwiyah Point rock fall, Yosemite National Park, USA. Earth Surface Processes and Landforms, 37(6), 680-691. https://doi.org/10.1002/ esp. 3206 